\title{
On the spectrum of closed $\mathrm{k}=2$ flux tubes in $\mathrm{D}=2+1 \mathrm{SU}(\mathrm{N})$ gauge theories
}

\author{
Andreas Athenodorou $^{a}$, Barak Bringoltz ${ }^{b}$ and Michael Teper ${ }^{a}$ \\ ${ }^{a}$ Rudolf Peierls Centre for Theoretical Physics, University of Oxford, \\ 1 Keble Road, Oxford OX1 3NP, UK \\ ${ }^{b}$ Department of Physics, University of Washington, Seattle, WA 98195-1560, USA
}

\begin{abstract}
We calculate the energy spectrum of a $k=2$ flux tube that is closed around a spatial torus, as a function of its length $l$. We do so for SU(4) and SU(5) gauge theories in 2 space dimensions. We find that to a very good approximation the eigenstates belong to the irreducible representations of the $\mathrm{SU}(N)$ group rather than just to its center, $Z_{N}$. We obtain convincing evidence that the low-lying states are, for $l$ not too small, very close to those of the Nambu-Goto free string theory (in flat space-time). The correction terms appear to be typically of $O(1)$ in appropriate units, much as one would expect if the bosonic string model were an effective string theory for the dynamics of these flux tubes. This is in marked contrast to the case of fundamental flux tubes where such corrections have been found to be unnaturally small. Moreover we find that these corrections appear to be particularly small when the 'phonons' along the string have the same momentum, and large when their momentum is opposite. This provides information about the detailed nature of the interactions in the effective string theory. We have searched for, but not found, extra states that would arise from the excitation of the massive modes presumably associated with the non-trivial structure of the flux tube.
\end{abstract}




\section{Introduction}

Consider a $D=2+1 \mathrm{SU}(N)$ gauge theory on a three-torus, and a loop of confining flux that winds once around a spatial torus of length $l$. If this flux is in the fundamental representation then one discovers [1, 2], that the low-lying energy spectrum of such a loop is the same as that of a corresponding closed string in the Nambu-Goto free string theory (in flat $D=2+1$ space-time) up to very small corrections, and that this is so even when $l$ is not much larger than the critical length at which the flux loop dissolves. This type of calculation is intended to provide detailed information about the effective string theory describing the gauge theory and should be useful for theoretical approaches from the string side.

In the usual field-theoretic picture of the confining string as some non-Abelian version of the flux tube in a (dual) superconductor, with some complicated internal spatial structure which should provide a complex set of internal modes to excite, it is not easy to understand how a closed flux tube that is not much longer than it is wide, can display the phonon-like excitations of a thin string to such a good accuracy. It is therefore tempting to see this as evidence for some underlying duality where the starting point in describing a flux tube is always a thin string, irrespective of its length, just as one has in the usual gauge/gravity duality. (See [3] for some recent reviews.) Even in the latter framework, however, one does expect states in the spectrum that are additional to those of the free bosonic string and which arise, for example, from the non-trivial background metric near the 'horizon' which leads to linear confinement. Finding such extra states would provide a useful clue about the nature of the hypothetical dual theory. So far no such 'extra' states have been identified in our lattice calculations [2] but this may be because the basis of operators we use in our variational calculation has little overlap onto any such states (as discussed below). This is one reason why it is useful to explore the spectrum of the quite different flux tubes that we study in this paper.

In this paper we explore what happens to the spectrum of a winding flux tube when the flux is in a higher representation than the fundamental. In particular for $N>3$ there exist new, completely stable flux tubes called $k$-strings. A $k$-string is a flux tube that emanates from a source which transforms as $\psi \rightarrow z^{k} \psi$ under a global gauge transformation belonging to the center of the group: $z \in Z_{N}$. One may think of this source as being a composite of $k$ fundamental sources, together with any number of adjoint sources which do not feel the centre. (It is clear that $k$ and $k^{\prime}=N-k$ are the 'same' and only $k \leq N / 2$ is interesting.) Since $k$ is unchanged by screening with gluons, the lightest flux tube in this sector will be stable. That is what one usually calls a $k$-string. (Sometimes we shall refer more loosely to all such flux tubes, stable and unstable, as $k$-strings.) While such a stable flux tube is of especial interest, excitations thereof, including flux tubes in other representations, will become stable at larger $N$ and are also of interest.

Given that a single fundamental flux loop is so well described by a free bosonic string theory, one might have expected that a $k$-string would simply look like some linear combination of free fundamental $(k=1)$ strings. So, for example, a $k=2$ string might look like a combination of a $k=1$ string that winds twice around the torus, and a state consisting of two $k=1$ strings, each winding once around the torus. The free string theory would, in this 
way, predict a simple relation between the string tensions: $\sigma_{k}=k \sigma_{f}$. However it has been known for some time [4] that the lightest $k$-string is in fact strongly bound (for $k / N$ not too small) and only acquires the free-string value at $N=\infty$ (as it must, at fixed $k$ ). Given that the binding is strong, e.g. in $\mathrm{SU}(4)$ one finds $\sigma_{k=2} \simeq 1.34 \sigma_{f} \ll 2 \sigma_{f}$, one can ask to what extent such a flux tube also behaves like a free bosonic string and what the corrections to such a behaviour tell us about the dynamics. This binding cannot be readily incorporated into the usual analytic frameworks [9, 12] since it presumably involves the exchange of small contractible loops of string (glueballs) that are not under analytic control. Thus such flux tubes are of particular interest.

The ground state energy of a closed $k=2$ flux tube of length $l$ has been calculated in [5], where it was found to be accurately described by the free Nambu-Goto string, with string tension $\sigma=\sigma_{k=2}$, and with corrections (in inverse powers of $1 / \sigma l^{2}$ ) that have coefficients that are $O(1)$ in natural units. This is what one would expect if the $k=2$ flux tube were to have an effective bosonic string description at large distances, and it provides a contrast with the fundamental flux tube where the corrections to the free string description are very much smaller [1, 2]. In practice, however, the ground state provides only a limited insight into the nature of the string spectrum and so, just as for the fundamental string, it is important to perform accurate calculations of at least a few excited states. This is difficult in a lattice calculation, where one can only hope to obtain accurate energies for those states which have a very high overlap onto the basis that one is using. For the fundamental flux loop we attacked this problem, with apparent success, by using a very large basis of operators [2]. Here we shall do the same for the $k=2$ spectrum, using the basis described in Section 2 and in Appendix A. Since we have seen from earlier calculations of this type [1, 2, 5, 6] that lattice spacing corrections are small, we shall not perform a continuum extrapolation, but will simply choose to perform our calculations at fixed, small values of the lattice spacing $a$. We shall perform calculations in $\mathrm{SU}(4)$ and $\mathrm{SU}(5)$ at $\beta=50$ and $\beta=80$ respectively.

In the next section we briefly describe the standard aspects of our lattice calculation and then, in some more detail, our choice of operators for winding flux tubes. We follow that up with a summary of the corresponding free string Nambu-Goto spectrum, since that will play an important part in the interpretation of our results. We then describe our results for the spectrum and find that we can interpret at least some of the states within the Nambu-Goto picture, simply on the basis of the way the energy depends on the length. We then turn to the question of whether the flux tubes know about the full $\mathrm{SU}(N)$ group or just about the $Z_{N}$ center and demonstrate that in fact it is the former. We then return to the very interesting question of whether the excited states without an obvious Nambu-Goto interpretation might not be revealing the excitation of new, internal degrees of freedom. To address this question we develop a heuristic method for comparing the shapes of the $k=2$ states with the $k=1$ states. This study leads us to infer that all the $k=2$ energy eigenstates over which we do have control, are most likely to be Nambu-Goto states with, in some cases, large corrections to the energy dependence. Comparing those states for which such corrections are small, with those for which they are large, enables us to say something qualitative and striking about where these corrections arise in the effective string theory. We end with a summary and our conclusions. 


\section{Methodology}

Our lattice framework is standard. We shall therefore be brief, except with those aspects that are important for understanding the statistical and systematic errors of the present calculation.

\section{1 lattice field theory}

We work on periodic cubic $L_{x} \times L_{y} \times L_{t}$ lattices with lattice spacing $a$. The degrees of freedom are $\mathrm{SU}(N)$ matrices, $U_{l}$, assigned to the links $l$ of the lattice. Our partition function is

$$
\mathcal{Z}(\beta)=\int \prod_{l} d U_{l} e^{-\beta \sum_{p}\left\{1-\frac{1}{N} \operatorname{Re} \operatorname{Tr} U_{p}\right\}}
$$

where $U_{p}$ is the ordered product of matrices around the boundary of the elementary square (plaquette) labelled by $p$. The continuum limit is approached by tuning $\beta \rightarrow \infty$ and in that limit $\beta=2 N / a g^{2}$ where $g^{2}$ is the usual continuum coupling, which has dimensions of mass in $D=2+1$. Our numerical calculations use a standard heat bath/over-relaxation Monte Carlo, where one updates all the $\mathrm{SU}(2)$ subgroups of the $\mathrm{SU}(N)$ matrices.

\section{2 calculating energies}

We typically wish to calculate the energy spectrum of some sector of states with particular quantum numbers (spin, momentum, parity, ...). We do so from correlation functions of operators $\phi(t)$ with those same quantum numbers. In a gauge-invariant calculation, the elementary components of such operators are typically traces of closed loops, where contractible loops typically project onto glueballs and non-contractible (winding) loops typically project onto winding loops of flux. In general we have

$$
C(t) \equiv\left\langle\phi^{\dagger}(t) \phi(0)\right\rangle=\sum_{n=0}|\langle n|\phi(0)| v a c\rangle|^{2} e^{-E_{n} t} \stackrel{t \rightarrow \infty}{\longrightarrow}|\langle 0|\phi(0)| v a c\rangle|^{2} e^{-E_{0} t} \quad ; \quad E_{i} \leq E_{i+1}
$$

where on a lattice we write $t=a n_{t}$, so we see that all the energies will be obtained in lattice units, as $a E_{i}$ ( $n_{t}$ is the separation in the Euclidean time direction in lattice units). Clearly, given infinite accuracy one could calculate the whole spectrum from one such correlation function. (Apart from states with accidentally zero overlap.) In the real world of finite accuracy, however, extracting more than the ground state energy, $E_{0}$, is an ill-conditioned problem so we shall proceed by the standard variational calculation described later on.

To obtain $E_{0}$ from a numerical calculation of $C(t)$, we perform a fit with a single exponential to $C(t)$ for $t \geq t_{1}$ and choose for $t_{1}$ the lowest value for which we obtain an acceptable fit. This is a way to estimate the asymptotic exponential decay in eqn(2). Now, since the fluctuations that determine the error in the Monte Carlo calculation of $C(t)$ are themselves proportional to a higher order correlation function which can be seen to possess a disconnected piece, the error is approximately independent of $t$, whereas $C(t)$, as we see from eqn(2), decreases at least exponentially with $t$. Thus the error/signal ratio increases exponentially with 
$t$ and one can only hope to obtain $E_{0}$ accurately if the corresponding ground state already dominates $C(t)$ at small values of $t$, i.e. one needs operators $\phi$ that have a large projection onto the desired ground state. This can be achieved using standard smearing/blocking techniques (see e.g. [6, 7]) which produce operators that are smooth on physical length scales, with little computational effort. If $a E_{0}$ is small enough that the error/signal ratio is small for a nontrivial range of $t=a n_{t} \geq t_{1}$, then the success of a single exponential fit provides significant evidence for it being appropriate. If however $a E_{0}$ is so large that this range is small, then we lose such evidence, and typically we will be using a single exponential fit over a range of $t$ where the contribution of higher excited states may still be significant. Thus our estimate of $a E_{0}$ will tend to become too high as $a E_{0}$ increases. This is an important sytematic error that will be visible in some of our results - in particular when we consider very long flux tubes (which are very massive) or highly excited flux tubes, even when they are not very long.

To calculate the excited states as well as the ground states, we carry out the following variational calculation. Consider a set of $n$ different smeared/blocked operators, $\left\{\phi_{i}(t) ; i=\right.$ $0, \ldots, n-1\}$, with the desired quantum numbers, and which have been normalised (but are not in general orthogonal). Now find the normalised linear combination of these operators, $\phi(t)=\Phi_{0}(t)$, that maximises $C\left(t=t_{0}\right)=\left\langle\phi^{\dagger}\left(t_{0}\right) \phi(0)\right\rangle=\left\langle\phi^{\dagger}(0)\left|\exp \left\{-H t_{0}\right\}\right| \phi(0)\right\rangle$ where $t_{0}$ is chosen small enough that all the correlators are accurately determined. (In practice we choose $t_{0}=a$.) Then $\Phi_{0}$ provides our best estimate of the ground state wavefunctional. Now consider that part of the basis $\left\{\phi_{i}\right\}$ that is orthogonal to $\Phi_{0}$, and find the operator $\phi(t)=\Phi_{1}(t)$ that once again maximises $C\left(t=t_{0}\right)=\left\langle\phi^{\dagger}(0)\left|\exp \left\{-H t_{0}\right\}\right| \phi(0)\right\rangle$ but now over this reduced basis. Then $\Phi_{1}$ provides our best estimate of the first excited wavefunctional. Repeating this procedure we obtain a set of operators, $\left\{\Phi_{i} ; i=0,1, \ldots\right\}$, that are our variational approximation to the exact energy wavefunctionals, $\left\{\Psi_{i}\right\}$. For each $\Phi_{i}$ we now consider the correlation function $\left\langle\Phi_{i}^{\dagger}(t) \Phi_{i}(0)\right\rangle$, perform single exponential fits as described above for the ground state, and extract an estimate of $a E_{i}$.

As for the ground state, it is critical that one has good overlaps, and in practice that means that we need $\left|\left\langle\Psi_{i}^{\dagger}(0) \Phi_{i}(0)\right\rangle\right|^{2} \geq 0.9$. To achieve this for several excited states we need a large basis of operators. In practice we use $\mathrm{O}(200)$ operators, as described below. For excited states there are additional systematic errors. First, the excited state may be unstable, e.g. if $\left(E_{i}-E_{0}\right)$ is greater than the lightest scalar glueball mass. This will not lead to any visible effects in our calculation as long as the decay width is small. This will be the case at large $N$, where decay widths vanish, but even in $\mathrm{SU}(3)$ they are usually too small to be important. (Hence the difficulty in observing string breaking directly in lattice QCD.) Another systematic error arises from the fact that an excited trial operator, $\Phi_{i}$, may have a non-zero overlap onto a lighter eigenstate, i.e. $\left|\left\langle\Psi_{j}^{\dagger}(0) \Phi_{i}(0)\right\rangle\right|^{2} \neq 0$ for $j<i$. If this overlap is small then it is only at large $t$ that $\left\langle\Phi_{i}^{\dagger}(t) \Phi_{i}(0)\right\rangle$ will behave like $\propto \exp \left\{-E_{j} t\right\}$ and there will be an intermediate range of $t$ where it will behave like $\propto \exp \left\{-E_{i} t\right\}$, and where one can extract $E_{i}$. However as this overlap becomes more significant, the ambiguity in extracting $E_{i}$ obviously will become larger. Another systematic error arises from the fact that the excited states show near-degeneracies that can be distorted by mixing effects which can be enhanced and complicated by the effects 
of statistical fluctuations in our variational calculation. 1 For all these reasons we shall confine ourselves to discussing only the lightest few excited states.

\section{3 winding flux loops}

Consider a loop of fundamental flux that winds once around the $x$-torus, so that it is of length $l=a L_{x}$. A generic operator $\phi_{l}$ that couples to such a periodic flux loop is the trace of an ordered product of link matrices, $l_{p}$, along a space-like curve $p$ that winds once around the $x$ torus, with the matrices in the fundamental representation. (This is an example of a Polyakov loop, or Wilson line, which may be in a general representation.) If we multiply all the $U_{x}$ link matrices in some given $x$-slice of the 3 -volume by an element $z \in Z_{N}$, then $l_{p} \rightarrow z l_{p}$. This center symmetry keeps track of the net winding: a $k$-string will transform as $l_{p, k} \rightarrow z^{k} l_{p, k}$. We will now specialise to the $k=2$ strings which are the subject of this paper.

We will consider $k=2$ flux tubes of two kinds. First there is a flux tube that winds once around the torus but contains flux in a representation of $\mathrm{SU}(N)$ that arises from the product of 2 fundamentals and any number of adjoints. The trace of a Wilson line in such a representation can in general be expressed in terms of a sum of operators that involve traces of powers and powers of traces of $l_{p}$ and of $l_{p}^{\dagger}$, such that the net power of $l_{p}$ minus the net power of $l_{p}^{\dagger}$ is 2 . In practice we shall limit ourselves to the operators $\operatorname{Tr} l_{p}^{2}$ and $\left\{\operatorname{Tr} l_{p}\right\}^{2}$ whose linear combinations correspond to the totally antisymmetric $(k=2 A)$ and totally symmetric $(k=2 S)$ representations

$$
\Phi_{2 A}=\operatorname{Tr} l_{p}^{2}-\left\{\operatorname{Tr} l_{p}\right\}^{2} ; \quad \Phi_{2 S}=\operatorname{Tr} l_{p}^{2}+\left\{\operatorname{Tr} l_{p}\right\}^{2}
$$

Since a $k=2 S$ source can become a $k=2 A$ source through gluon screening, there is no reason for such operators to be orthogonal or to assume that such $\mathrm{SU}(N)$ representations will be useful in labelling the actual eigenstates. Whether they are useful is a dynamical question that we shall determine in this paper. The second type of $k=2$ flux tube that we consider is one that carries fundamental flux and winds twice around the torus. A typical trial operator for such a flux tube will be

$$
\Phi_{w=2}=\operatorname{Tr}\left\{l_{p} l_{p^{\prime}}\right\}
$$

where the two single-winding paths $p$ and $p^{\prime}$ are in general not the same but the joint path $\tilde{p}=p p^{\prime}$ is continuous and periodic with a period $2 l_{x}$. Note that the $w=2$ operators need not be orthogonal to the $k=2 A$ and $k=2 S$ operators. Indeed because of the strong $k=2$ binding, one might expect that if $p$ and $p^{\prime}$ are similar, so that the second winding of the flux tube is physically close to the first winding, the attractive interactions will give a significant probability of binding into a singly-wound $k=2$ flux tube, and this will be reflected in the overlaps of the corresponding operators. One has therefore the heuristic expectation that to see a genuine $w=2$ flux tube one needs to use very different paths $p$ and $p^{\prime}$.

In order to obtain good overlaps onto the true energy eigenstates, we use smeared/blocked links in our construction of $l_{p}$. Usually, and always when it matters, as in the projection onto

\footnotetext{
${ }^{1}$ For example, a correlation between two nearly-degenerate states, that would average to zero with infinite statistics, can induce an artificial level repulsion.
} 
specific representations of the flux, we make sure that our blocked matrices are all $\mathrm{SU}(N)$. (For historic reasons this is not always the case for some of our oldest calculations, although this will not affect any conclusions we shall draw from them.) Using operators with different levels of smearing probes different transverse size scales, and this forms part of our variational basis.

What are the other quantum numbers that label such a flux tube? First, we recall that in 2 space dimensions, there are no rotations around the axis of the tube and in the confining phase a flux tube that is rotated by $\pi / 2$, so that it winds around the $y$-torus, will have no overlap with one winding around the $x$-torus, so this symmetry is uninteresting. We do however have translations and corresponding momenta. We project onto zero-momentum transverse to the flux tube, $p_{y}=0$, by adding all $y$-translations of our basic operator. We do not consider $p_{y} \neq 0$ since previous calculations have shown that for the range of lattice spacings we shall consider, the continuum energy-momentum dispersion relation is accurately satisfied and so there does not seem to be anything new that one might learn using non-zero transverse momenta. More interesting is the momentum along the flux tube, $p_{x}=2 \pi q / l$. Here we translate the loop by $x_{0}$ in the $x$ direction, multiply it by $\exp \left\{i p_{x} x_{0}\right\}$, and sum over all such translations. Clearly if the flux tube is invariant under translations along its axis, as we might expect if it is in its ground state, then imposing $q \neq 0$ in this way will produce a null state. To have $q \neq 0$ the state must be excited in some way. Thus calculating the spectrum for various $q \neq 0$ promises to teach us something new. Another symmetry is (two dimensional) parity, P. Again one expects that to have $P=-$ one requires a deformation of the flux tube, i.e. an 'excitation', that is different under reflection. Finally there is charge conjugation $C$. This operation changes the direction of the arrow, $l_{p} \rightarrow l_{p}^{\dagger}$, so that $\operatorname{Re} \phi$ is $C=+$ and $\operatorname{Im} \phi$ is $C=-$. Since $l_{p} \rightarrow z l_{p}$ and $l_{p}^{\dagger} \rightarrow z^{\dagger} l_{p}^{\dagger}$ under the center transformation introduced earlier, there is in general zero overlap between $l_{p}$ and $l_{p}^{\dagger}$ so the $C=+$ and $C=-$ sectors are degenerate and the label is therefore uninteresting. However this is not the case when $k=N / 2$. In that case there can be mixing and the $C= \pm$ sectors need not be degenerate. In our case, that occurs for $k=2$ in $\mathrm{SU}(4)$. However it is easy to see that, for $S U(4), \operatorname{Im} \Phi_{k=2 A} \equiv 0$. Thus to the extent that there is a $k=2 A$ sector of states, we will only have $C=+$ therein. For $k=2 S$ on the other hand, $C= \pm$ can be a useful label in $\mathrm{SU}(4)$.

In Appendix A we describe in more detail the construction of the actual operators we use.

\section{$3 \quad$ Flux tubes as strings}

The immediate goal of our calculations is to provide information about the spectrum of $k$ strings that will complement what we have learned previously about fundamental $k=1$ flux tubes. Our wider purpose, however, is to provide useful information for theoretical approaches to understanding confinement and the dynamics of QCD. Thus a central step in our analysis is to compare our results to what one might expect on very general grounds, so as to pin-point what might be new and interesting. In this section we shall first discuss what these general expectations might be, and then we summarise the detailed spectrum of the free Nambu-Goto string theory (in flat space-time) since this turns out to provide a very useful comparative 
benchmark.

The flux tubes that will interest us here, are those that are wound around a spatial $x$-torus with all other space-time dimensions effectively infinite. As we shrink the length of this torus, $l=a L_{x}$, such a system will undergo a phase transition at a critical value $l=l_{c}=1 / T_{c}$ where $T_{c}$ is the deconfining temperature. For $l \leq l_{c}$ the ground state is no longer a flux tube, but rather has zero energy. In $D=2+1$ and for $N \geq 4$ one finds $l_{c} \sqrt{ } \sigma_{f} \simeq 1.1$ [8]. Thus if we want to discuss closed flux tubes, we must choose $l>l_{c}$.

\section{1 general expectations}

Consider a flux tube of length $l=a L_{x}$ winding around the $x$-torus. Translations leave its energy unchanged. However under transverse translations the state is not the same: the choice of a position for the flux tube breaks this symmetry spontaneously and we expect to have corresponding massless fluctuations in the excitation spectrum of the flux tube. Since we would expect the ground state to be invariant under translations along the flux tube, we do not expect this longitudinal translation symmetry to be spontaneously broken and we do not expect any further Goldstone bosons from that source.

Now, since the $\mathrm{SU}(N)$ field theory has only one scale, say $l_{\sigma}=1 / \sqrt{\sigma_{f}}$, the width of the flux tube should in general be on that scale. On length scales $\gg l_{\sigma}$, and for excitation energies $\ll 1 / l_{\sigma}$ the flux tube should effectively behave as a thin string. The classical fluctuations of such a periodic string have wavelength $\lambda_{n}=l / n$ and hence frequency $\omega_{n}=2 \pi n / l$. The corresponding oscillators will be quantised, and the excitations can be thought of as made up out of phonons of energy $\omega_{n}$ and with momenta $p_{n}= \pm \omega_{n}$ along the string. This is the expected Goldstone boson, which is massless and can have any momentum allowed by periodicity. At large $l$ all these eigenstates converge

$$
\lim _{l \rightarrow \infty} E_{n} / \sigma l=1, \quad \forall n
$$

At finite $l$ there will be shift in the ground-state energy from the zero-point energies of the oscillators (essentially a Casimir energy) and this leads to the well-known Luscher correction [9] to the string energy:

$$
E_{0}(l) \stackrel{l \rightarrow \infty}{=} \sigma l\left(1-\frac{\pi}{6} \frac{D-2}{\sigma l^{2}}\right)
$$

which reflects the asymptotic low-lying $n=0$ excitation spectrum

$$
E_{n}(l) \stackrel{l \rightarrow \infty}{=} \sigma l\left(1+\frac{4 \pi}{\sigma l^{2}}\left(n-\frac{D-2}{24}\right)\right)
$$

The coefficient of the $O\left(1 / \sigma l^{2}\right)$ term is universal and the value shown in eqn(6],7) is the one for the simple bosonic string universality class, where the only massless excitations of the flux tube arise from the spontaneously broken transverse translation invariance. There is strong numerical support for this choice in the case of both fundamental and $k=2$ flux tubes [1, 5], and this is of interest since in principle there might have been other, less obvious, massless fluctuations that would have led to a different coefficient. 
The large- $l$ expansion in powers of $1 / \sigma l^{2}$, that we see in eqns(6],7), arises naturally in the classic framework of Luscher, Symanzik and Weisz, [9, 10] where one categorises and constrains the possible interaction terms in the transverse displacement fields. These interaction terms will in general lead to non-trivial interactions amongst the phonons and are encoded in the higher order corrections to the ground and excited state energies shown in eqns(6]7). This approach has recently led to the remarkable conclusion [10] that the next term in this expansion is $\propto 1 /\left(\sigma l^{2}\right)^{2}$ with the same coefficient as in the Nambu-Goto spectrum (see below). A similar conclusion was independently reached in [11] using the alternative Polchinski-Strominger framework [12] where one builds up an effective D-dimensional string theory within a general string path-integral setting.

These two theoretical approaches have in common that they only hope to treat very long flux tubes and, in effect, only eigenstates that are string-like in the sense of eqn(馬). However it is common to think of the confining flux tube as being qualitatively similar to a NielsenOlesen vortex, albeit one that is a non-Abelian dual-superconducter. Such a vortex will in general also have a pattern of excitations that reflects the effective theory producing its non-trivial structure. (For an explicit example in related, more tractable theories see [13].) These excitations will typically be massive, leading to string eigenstates with energies that will typically be 'much' larger than those of stringy excitations at large enough $l$ :

$$
\tilde{E}(l) \simeq E_{0}(l)+\mu \stackrel{l \rightarrow \infty}{>} E_{n}(l) \simeq \sigma l+\frac{4 \pi}{l}\left(n-\frac{D-2}{24}\right)+\ldots
$$

One's first guess for $\mu$ might be something on the order of the lightest glueball mass, or the splitting between that state and its first excitation. Using values for the lightest glueball masses from [6] this would suggest

$$
\mu \sim 2-4 \sqrt{ } \sigma_{f}
$$

Such states should become particularly visible at smaller values of $l$ where the gap between the stringy states becomes larger than this estimate for $\mu$.

One might simply give up on describing such massive excitations in the belief that the spectrum of anything but a very long flux tube will be too complex to be theoretically tractable. However our earlier calculations of the low-lying excitations of the fundamental flux loop have revealed that the simple stringy predictions of the free bosonic Nambu-Goto string theory hold very accurately even for flux tubes as short as $l \sqrt{\sigma_{f}} \simeq 1.5$ [1, 2]. That a flux tube which is only a little longer than it is wide should display simple string-like excitation modes is not something that one can understand within a generic vortex picture. It suggests that despite appearances short flux tubes do have a stringy description. Does this mean that the flux tube is indeed a thin string, with its apparent width simply a manifestation of the zero-point fluctuations described above? This would immediately come up against the difficulties of defining a consistent bosonic string theory in 3 or 4 dimensions. A more interesting possibility is offered by a dual gravity description of the AdS/CFT type. (In fact non-CFT and possibly non-AdS.) Here the flux loop is indeed always described by a string but now this string is in a geometry that is, at least initially, 10 dimensional. Of course the spectrum is now that of a Nambu-Goto string in curved rather than flat space-time, and this curvature is crucial to providing linear 
confinement rather than a conformal-like Coulomb interaction. In this picture it may not be unnatural for some stringy states to arise even for short strings, and the massive modes are of great interest as they provide clues as to the geometry of the dual theory.

\subsection{Nambu-Goto closed string spectrum}

The simplest specific bosonic string theory is the free string theory: Nambu-Goto in flat spacetime. Of course such a theory can only be consistently formulated in 26 dimensions. However, as discussed by Polchinski and Strominger [12], if one is interested in an effective string theory for single long strings, then the Weyl anomaly does not pose an obstacle to working in $D<26$. (As was already pointed out long ago by Olesen [14].) The spectrum of the Nambu-Goto free bosonic string theory in $D=2+1$ or $D=3+1$ and in the sector of a single long string, has also long been known [15, 16]. We shall briefly summarise this spectrum here, since it will provide our central point of comparison in this paper.

Consider a single closed string that wraps $w$ times around a spatial torus. The free string spectrum corresponds to quantised oscillations of the string that may be thought of as phonon modes traveling clockwise (right-handed) and anticlockwise (left-handed) along the background string. Our convention is to take the momenta of the latter as positive and of the former as negative. The modes are massless, corresponding to the Goldstone bosons that arise from the fact that the presence of the background string spontaneously breaks the transverse translation invariance. So the energy of a phonon equals the absolute value of its momentum. This momentum is along the string and is constrained by the latter's periodicity to be $\pm 2 \pi k / l w$ where $k=0,1,2, \ldots$ and $l w=a L_{x} w$ is the length of the string. Of course, the total momentum is constrained to be $2 \pi q / l \equiv 2 \pi q w / l w$, with $q=0, \pm 1, \pm 2, \ldots$, since the Hamiltonian is defined on a spatial torus of size $l$ (and not $l w$ ).

Let $n_{L}(k), n_{R}\left(k^{\prime}\right)$ be the number of left and right handed phonons with momentum $2 \pi k / l w$ and $-2 \pi k^{\prime} / l w$ respectively. A free string state is defined by the total set of phonons and the total energy and momentum will be determined by

$$
N_{L}=\sum_{k} \sum_{n_{L}(k)} n_{L}(k) k, \quad N_{R}=\sum_{k^{\prime}} \sum_{n_{R}\left(k^{\prime}\right)} n_{R}\left(k^{\prime}\right) k^{\prime},
$$

The sum of the phonon momenta is just the total momentum of the excited string and is therefore constrained to be

$$
N_{L}-N_{R}=q w .
$$

In addition the states have a parity, $P$. Under $P$ (in two space dimensions) the transverse displacement changes sign, so the corresponding creation operators for these displacements, the right-handed and left-handed phonon operators, will change sign. That is to say,

$$
P=(-1)^{\text {number of phonons }} \equiv(-1)^{\sum_{k} \sum_{n_{L}(k)} n_{L}(k)+\sum_{k^{\prime}} \sum_{n_{R}\left(k^{\prime}\right)} n_{R}\left(k^{\prime}\right)} .
$$

Writing down the 4-momentum of such a state and taking its scalar product, one obtains the energy-squared:

$$
E_{N_{L}, N_{R}, q, w}^{2}=(\sigma l w)^{2}+8 \pi \sigma\left(\frac{N_{L}+N_{R}}{2}-\frac{D-2}{24}\right)+\left(\frac{2 \pi q}{l}\right)^{2}
$$


where the negative piece in the middle term arises from the zero-point energies of the oscillators. These energy levels will have a degeneracy that is determined by the number of different ways one can produce the same value of $N_{L}+N_{R}$ in eqn(10). As an example we present in Table [1] the seven lowest energy levels of a closed bosonic string that winds once around the torus for $q=0,1,2$, in terms of the left and right movers (creation operators) $\alpha_{-k}$ and $\bar{\alpha}_{-k^{\prime}}$.

\section{3 corrections to the free string}

We now consider how to incorporate corrections to the free string spectrum. We consider $q=0$ and $w=1$ but the extension to other values should be obvious.

The conventional Luscher analysis [9, 10] of confining strings focuses on the effective Lagrangian for the displacement field, and categorises the interactions in powers of these fields and derivatives. Originally this showed that in an expansion of $E(l)$ in powers of $1 / \sigma l^{2}$, the first correction, which arises from the oscillator zero-point energies, is universal and is proportional to the string central charge. Given the universality it is no surprise that this term, the well-known Luscher correction, is precisely what one obtains as a first correction to $\sigma l$ when one expands the Nambu-Goto expression for $E(l)$ in eqn(13) in powers of $1 / \sigma l^{2}$. More recently [10] this analysis has been carried further, and it has been shown, on quite general grounds, that in $D=2+1$ the next term in this expansion also coincides with what one obtains from Nambu-Goto, i.e.

$$
E_{n}=\sigma l+\frac{4 \pi}{l}\left(n-\frac{1}{24}\right)-\frac{8 \pi^{2}}{\sigma l^{3}}\left(n-\frac{1}{24}\right)^{2}+\mathcal{O}\left(1 / l^{4}\right),
$$

where $n=0,1,2 \ldots$. Quite independently, an extension [11] of the work of Polchinski and Strominger [12] reached the same conclusion (but for $D=3+1$ as well).

In our calculations of the $k=1$ flux loop [1, 2] we discovered that the actual spectrum is reproduced by the free string Nambu-Goto spectrum much better than one would expect from eqn(14). In fact there is even good agreement for values of $l$ that are so small that the expansion of eqn(13) in powers of $1 / \sigma l^{2}$ completely diverges. The implication is clear [2]: we should use the full Nambu-Goto expression as our starting point, and consider corrections to that. In that spirit we write

$$
E_{n}^{2}(l)=(\sigma l)^{2}+8 \pi \sigma\left(n-\frac{1}{24}\right)-\sigma \frac{C_{p}}{(l \sqrt{\sigma})^{p}}, \quad p \geq 3
$$

where $p=3$ corresponds to the $\mathcal{O}\left(1 / l^{4}\right)$ correction in eqn(14). In this paper we will fit our $k=2$ spectra with eqn(15), with the leading permitted correction of $p=3$.

\section{Spectrum of $k=2$ flux tubes}

We have performed calculations in $\mathrm{SU}(4)$ and $\mathrm{SU}(5)$ at $\beta=50$ and $\beta=80$ respectively. Since $\beta=2 N^{2} / \lambda$, where $\lambda=g^{2} N$ is the 't Hooft coupling, these values of $\beta$ should correspond to 
roughly the same value of the lattice spacing $a$, and indeed we find

$$
a \sqrt{ } \sigma_{f}=\left\{\begin{array}{lll}
0.1310(2) & : & S U(4), \beta=50 \\
0.1298(1) & : & S U(5), \beta=80
\end{array}\right.
$$

Extensive experience with glueball masses [6] the deconfinement temperature [8] and the spectrum of fundamental strings [1, 2] indicates that lattice spacing corrections for such values of $a$ are negligible and we shall therefore assume that what we obtain is indeed continuum physics.

We calculate the $k=2$ spectra of flux tubes winding around $x$-tori that range in length from $l=12 a$ to $l=32 a$. As we see from eqn(16) this corresponds to $l \sqrt{ } \sigma_{f} \in[1.56,4.20]$. Thus our shortest flux tubes are presumably not much longer than they are wide - hardly 'tubes' at all. For comparison, the minimum length $l_{c}$ below which we lose such flux tubes completely is 8 ]

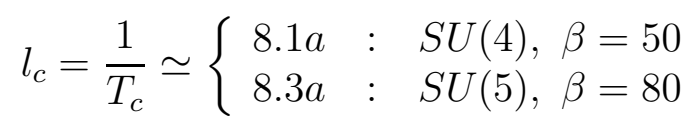

Since we are interested in accurately identifying corrections to the asymptotic linear variation with $l$, it is important that we minimise systematic errors that vary with $l$. Thus at small $l$ we must make $L_{t}$ large enough so that the contribution of both $k=1$ and $k=2$ loops to the partition function is negligible, and all our energies are normalised to the correct vacuum energy. Similarly we must also make the transverse size $L_{y}$ large enough to avoid finite size corrections. Thus, following a variety of checks, the lattices we use for SU(5) are: $12 \times 32 \times 80$, $16 \times 32 \times 40,20 \times 24 \times 32,24 \times 24 \times 32,28 \times 28 \times 32,32 \times 32 \times 32$. For our SU(4) calculations, which were performed earlier, we use slightly smaller lattices, where finite volume corrections, for the smallest values of $l$ could be non-negligible: $12 \times 24 \times 40,16 \times 24 \times 40,20 \times 24 \times 32$, $24 \times 24 \times 32,28 \times 28 \times 32,32 \times 32 \times 32$. We typically perform 500,000 Monte Carlo sweeps and measure the full correlation matrix every 5 sweeps.

As described earlier, the relevant quantum numbers in labelling our $k=2$ flux tubes are the momentum, $q$, along the flux tube and the parity $P$. We ignore charge conjugation since it is only relevant for $N=4$, and then only for the heavier $k=2 S$ states. In addition, the flux may be in different representations of $\mathrm{SU}(N)$. The $S U(N)$ representation is, however, not a conserved quantum number since it can change under screening by gluons. Nonetheless we shall see later that the mixing between $k=2 A$ and $k=2 S$ states is typically very small, and so in our analysis in this section we shall categorise the states in this way as well. (Our basis of operators does not allow us to explore other representations.) Note that where it significantly improves the calculation we use our full $k=2$ basis of operators, although we categorise the states by their dominant $k=2 A$ or $k=2 S$ component.

\section{$4.1 \quad k=2 A$}

In Figs. 1 and 2 we plot the energies of the lightest states with momenta $q=0,1,2$ along the flux tube, for $\mathrm{SU}(4)$ and $\mathrm{SU}(5)$ respectively. We observe that for all values of $l$ the lightest $q=0$ state has $P=+$, the lightest $q=1$ state has $P=-$, and for $q=2$ there are two 
nearly degenerate lightest states, one with $P=+$ and one with $P=-$. We note that these quantum numbers and (near-)degeneracies are precisely those of the Nambu-Goto free string theory. (See Table 1.) All these states have a projection onto $2 A$ that is very much larger than onto $2 S$ and we therefore regard them as constituting part of the $2 A$ spectrum. We have removed all lattice units by normalising the loop energies and the loop lengths to the value of $a \sqrt{ } \sigma_{f}$ that has been obtained from $k=1$ flux loops in the same calculation. (The errors on this quantity are much smaller than all our other errors.)

We fit the $q=0$ ground state using the Nambu-Goto expression with the leading permitted correction, as in eqn(15), [10, 11],

$$
E^{2}(l)=\left(\sigma_{2 A} l\right)^{2}\left(1-\frac{\pi}{3} \frac{1}{\sigma_{2 A} l^{2}}\right)-\sigma_{2 A} \frac{C_{3}}{\left\{\sqrt{\sigma_{2 A}} l\right\}^{3}}
$$

to obtain

$$
\begin{array}{ll}
a^{2} \sigma_{2 A}=0.023233(51)=1.354(5) a^{2} \sigma_{f} ; C_{3}=2.93(21) & : S U(4) \\
a^{2} \sigma_{2 A}=0.025825(50)=1.533(4) a^{2} \sigma_{f} ; C_{3}=2.28(22) & : S U(5)
\end{array}
$$

Note that the values of $\sigma_{2 A} / \sigma_{f}$ are close to the quadratic Casimir ratios, $k(N-k) /(N-1)$.

We observe that when expressed in terms of natural units, as in eqn(18), the correction to Nambu-Goto has a coefficient $C_{3} \sim O(1)$. This is a natural value if we regard the string theory as an effective theory, in the Nambu-Goto universality class. It is to be contrasted with the value $C_{3} \sim O(1 / 10)$ that one finds for the fundamental flux tube, and serves to emphasise how remarkably well the latter is described by the free bosonic Nambu-Goto string theory.

Using these calculated values of $\sigma_{2 A}$, we calculate the lightest $q=0,1,2$ energies of the Nambu-Goto model, and plot the results in Figs 1 and 2. We observe an excellent agreement between our numerical results and the Nambu-Goto values. Note that once the $q=0$ ground state has been fitted, the $q=1,2$ energies are parameter-free predictions.

Since the Nambu-Goto prediction appears to work so well, it is worth examining the results more closely to try and isolate how well the phonon excitation energy is being reproduced by our numerical results. To do this we note from eqn(13) that the piece of the energy that is due to the phonons is given by

$$
\Delta(q, l)=E_{g s}^{2}(q ; l)-E_{g s}^{2}(q=0 ; l)-\left(\frac{\pi q}{l}\right)^{2}
$$

Using our numerically calculated values for $E_{g s}(q ; l)$ we obtain the results displayed in Figs. 3 and 4 for $\mathrm{SU}(4)$ and $\mathrm{SU}(5)$ respectively. (We choose to use our numerical value for $E_{g s}(q=0 ; l)$ rather than the Nambu-Goto prediction since the former already has some small corrections which we would regard as belonging to the Casimir energy rather than to the excitation energy that we are trying to isolate here.) For comparison we show the Nambu-Goto phonon excitation energies as well. We observe that the simple free string excitation energies provide an excellent description of the string excitation energies over almost our whole range of string lengths, with significant deviations only beginning to appear at the smallest value of $l, l \sqrt{ } \sigma \simeq 1.5$. Given the qualitative (quantum numbers, degeneracies) and quantitative 
agreement between Nambu-Goto and our results, we will assume that these eigenstates are very close to those of the free string theory, with the corresponding phonon excitations.

We began this Section with the ground states for various $q$ because, as explained earlier, ground state energies can be extracted more reliably than excited state energies. We now turn to the excited states that form the low-lying $q=0, P=+$ spectrum. We plot our results for $\mathrm{SU}(5)$ in Fig. 6 together with the Nambu-Goto predictions. (The SU(4) results are essentially the same and are given in Fig. 5.) The first excited state shows substantial deviations from the free string energies, and the next two states even more so. However at least the former does seem to be approaching the free string energy as $l$ increases. This might tempt us to see it as a string excitation, but this would be dangerous, since we also observe that to quite a good approximation this first excited state is greater than the ground state by a constant value,

$$
E_{1}(q=0, l) \simeq E_{0}(q=0, l)+\epsilon \quad ; \quad \epsilon \simeq 2 \sqrt{ } \sigma_{f},
$$

which is reminiscent of our expectation for non-stringy states as described in eqns(8] (9). Such a state would cross the level of the first string excitation at some larger $l$, and perhaps that, not a convergence, is what we are seeing in Fig. 6. At this stage we clearly cannot decide how to classify this excited state. We shall return to this question in a later Section, where we shall find that looking directly at the wavefunctional will give us an unambiguous answer.

\section{$4.2 k=2 S$}

As we shall see later on, all the states we analyse appear to transform primarily as $k=2 \mathrm{~A}$ or as $k=2 S$ (or as $w=2$ fundamental). However the lowest $k=2 A$ states are much lighter than the corresponding $k=2 S$ states, so the latter will be much more susceptible to the systematic errors discussed in Section 2.2 - in particular to their masses being over-estimated.

Bearing this caveat in mind, we display in Figs. 7 and 8 the energies of the lightest $k=2 S$ states with momenta $q=0,1,2$, for $\mathrm{SU}(4)$ and $\mathrm{SU}(5)$ respectively. Qualitatively the picture is much as for the $k=2 A$ states: we find that for all values of $l$ the lightest $q=0$ state has $P=+$, the lightest $q=1$ state has $P=-$, and for $q=2$ there are two nearly degenerate lightest states, one with $P=+$ and one with $P=-$. All this fits in with the Nambu-Goto free string picture. Turning to a quantitative comparison with Nambu-Goto, we encounter an immediate difficulty. Our estimate of the $l=32$ ground state energy is too high to fit in with an $E(l)$ tending to an asymptotically linear behaviour. We therefore assume that what we are seeing here is the result of a systematic error of the kind described in Section 2.2, and which is perhaps twice the size of the statistical error. If we exclude this value from our fit to the $q=0$ ground state using eqn(13), we obtain

$$
\begin{array}{ll}
a^{2} \sigma_{2 S}=0.03957(45)=2.306(27) a^{2} \sigma_{f} & : S U(4) \\
a^{2} \sigma_{2 S}=0.03793(33)=2.251(20) a^{2} \sigma_{f} & : S U(5)
\end{array}
$$

(where the correction coefficients, $C_{3}$, are not well-determined). In fact if we had included the $l=32$ data point we would have obtained almost identical values of $\sigma_{2 S}$, but with a much 
worse $\chi^{2}$. We note once again that the values of $\sigma_{2 S} / \sigma_{f}$ are close to the quadratic Casimir ratios, in this case $k(N+k) /(N+1)$.

Using these values of $\sigma_{2 S}$, we show the corresponding $q=0,1,2$ ground state Nambu-Goto spectra in Figs. 7 and 8. Semi-quantitatively the agreement is good; however the longer, more massive states tend to overshoot the predicted values. It is plausible that this is again due to the systematic errors of our calculation growing with the calculated mass.

In Fig. 9] we extract the phonon excitation energies from our SU(5) values, using eqn(22)). (The SU(4) results are very similar.) There is good qualitative agreement with Nambu-Goto, but clearly we cannot say more than that.

In contrast to the $k=2 A$ case, we have severe difficulty in extracting excited state energies and hence do not attempt to produce a plot that is analogous to Fig. 6.

To conclude, it is probably fair to say that the $q=0,1,2$ ground states provide significant evidence that the $k=2 S$ flux tube also behaves like a free bosonic string, at least for these states, but that any attempt at a stronger statement is hampered by the current uncertainties of our numerical calculations for these very massive excited states.

\section{Group or centre?}

For each $k$, and for each set of conserved quantum numbers $P, C$ and $q$, there should only be one absolutely stable flux tube. (Except e.g. where it can decay into a string with the same $k$ but with some of the other quantum numbers different, and accompanied by glueballs that carry the compensating quantum numbers.) However, flux tubes carrying flux in an $S U(N)$ representation with $N$-ality $k$ that is, in general, not stable, can be stable enough to be readily identifiable in our calculations. If so it is an interesting question to ask if they are individually described by an effective bosonic string theory with an appropriate string tension. We have, of course, already answered this question in the affirmative in earlier sections, where we examined the $k=2$ flux tubes in the totally symmetric and antisymmetric representations. (Which are the only ones accessible to our limited choice of operators.) Here we will present evidence that the portion of the $k=2$ spectrum accessible to us is, to a good approximation, the sum of disjoint $k=2 A$ and $k=2 S$ spectra. This provides the promised continuation of the discussion of this question in [5] to which we refer the reader for further background.

\section{1 operator overlaps}

We begin by looking at the overlaps of our $k=2 A$ basis operators, $\left\{\Phi_{2 A}^{i}, i=0,1,2, \ldots\right\}$, onto their $k=2 S$ counterparts, $\left\{\Phi_{2 S}^{i}, i=0,1,2, \ldots\right\}$. For this purpose we define the normalised overlaps:

$$
O_{A S}(i, j ; t)=\frac{<\Phi_{2 A, i}^{\dagger}(t) \Phi_{2 S, j}(0)>}{<\Phi_{2 A, i}^{\dagger}(t) \Phi_{2 A, i}(0)>^{1 / 2}<\Phi_{2 S, j}^{\dagger}(t) \Phi_{2 S, j}(0)>^{1 / 2}}
$$

In Table 4 of [5] we presented results for such overlaps, at $t=0$, using a small basis of blocked/smeared Polyakov loops of length $l=24 a$ on a $24^{2} 32$ lattice in $\operatorname{SU}(5)$ at $\beta=80$ 
(corresponding to $l \sqrt{\sigma_{f}} \sim 3$ ). We found that most of the overlaps were zero within errors, except for those where at least one of the operators was at the largest blocking level; but even there the largest overlap was still a very small $\sim 0.03$. This provided some striking evidence that the theory does indeed break up into almost disjoint $k=2 A$ and $k=2 S$ sectors. However, while this small basis has a reasonably good overlap onto the $k=2 A$ and $k=2 S$ ground states, the overlap onto excited states is in general poor. It is therefore interesting to see what happens when we consider the much larger operator basis of the present paper, which has a good overlap onto most of the low-lying states. Another question is how much the results of [5] are affected by finite volume corrections. The point being that the only significant overlaps were for the largest 5'th blocking level, where the blocked link is of length $16 a$ and has a width that is at least as large - that is to say, the operator more-or-less covers the whole spatial torus. Finally a very interesting question is what happens for $t \neq 0$, since this probes more directly the represntation content of the energy eigenstates.

To address the question of finite volume corrections we perform some $t=0$ calculations in $\mathrm{SU}(4)$ at $\beta=32$. (Since in $\mathrm{SU}(4)$ the $k=2 A$ operators are necessarily $C=+$, we take their overlaps with the $C=+$ piece of the $k=2 S$ operators.) We start with $l=16 a$ because this also corresponds to $l \sqrt{\sigma_{f}} \sim 3$. Note that on a $16^{2}$ spatial lattice the 5 'th blocking level really does cover the whole spatial torus; so any finite size corrections should be larger than in [5]. We show a selection of the overlaps on a $16^{2} 20$ lattice in Table 2, including all those which are large enough to be significant. (Nearly all those not shown are zero within errors.) We see that a few overlaps are indeed not small, but that they again occur when one of the operators is at the largest blocking level. We now see what happens if we keep the same loop length, but vary the transverse spatial size, i.e. we work on $16 \times l_{y}$ spatial lattices where we vary $l_{y}$. What one finds is that the overlaps rapidly decrease with increasing $l_{y}$ (and rapidly increases as we decrease $l_{y}$ below $l$ ). We show examples in Table 2 for $l_{y}=20$ and $l_{y}=48$. The decrease is however not to zero but to a finite value; in fact the $l_{y}=48$ values are asymptotic within errors. These values are very small, and much smaller than on the original $16^{2}$ torus. We now turn to what happens as we increase $l$ while staying on an $l^{2}$ lattice. As we see in Table 2 this also strongly decreases the overlaps. In this case all the overlaps appear to tend to zero as $l$ increases. Of course higher blocking levels will become possible at larger $l$, and we would expect the largest of these to sometimes generate significant overlaps, but such operators will, eventually, have very small overlaps onto the low-lying flux loop states.

We now turn to the overlap between all the operators in our extended basis. These have been calculated on a $32^{3}$ lattice for $P=+, q=0$ and $\mathrm{SU}(4)$ at $\beta=50$. A Table is not practical, so we provide in Fig. 10 a three-dimensional plot of the values of $\left|O_{A S}(i, j ; t=0)\right|$ as $i$ and $j$ run over our basis of some 80 operators. As we might have expected from our more limited study above, the overlaps are mostly very small, and usually zero within the errors. Only those that involve operators at the very largest blocking levels are significant although even these are numerically small. (Here the 5'th blocking level is the largest, and it appears in our operator labeling at multiples of 5.) We can further assume, given what we found above, that most of these larger values are much enhanced by finite volume corrections. Thus this study confirms that even when we extend the basis so that it is quasi-complete for the low-lying spectrum of the $k=2$ flux tube, the near-orthogonality of the $k=2 A$ and 
$k=2 S$ sectors is maintained. Physically this suggests that the effects of screening $k=2 S$ down to $k=2 A$ are very weak, and why that should be so, even in $\mathrm{SU}(4)$, needs to be better understood.

Of course the near orthogonality of the $k=2 A$ and $k=2 S$ sectors does not necessarily imply that the energy eigenstates belong to one sector or the other (although it is a condition for that to be possible). To be sensitive to the eigenstate content we need to look at $\left|O_{A S}(i, j ; t)\right|$ for $t \neq 0$. Since the correlators decrease exponentially with $t$, we only have accurate results for the lowest values of $t$ and we will therefore restrict ourselves in the following dicussion to $t \leq 2$. Now, if the eigenstates were linear combinations of comparable amounts of $k=2 \mathrm{~A}$ and $k=2 S$, then we would expect to find $\left|O_{A S}(i, j ; t \neq 0)\right| \sim O(1)$. If on the other hand $\left|O_{A S}(i, j ; t \neq 0)\right|$ is 'small' then this suggests that the lightest states are largely in one sector or the other. To determine which is the case, we turn to our high statistics calculation on a $24^{2} 32$ lattice in $\mathrm{SU}(5)$ at $\beta=80$. In Table 3 we show the same selection of overlaps as in Table 2, but now for $t=0,1,2$. (The $t=0$ results differ from those shown in 5 because the statistics here are ten times greater.) We see that the $t \neq 0$ overlaps do indeed remain very small. Moreover some lower statistics calculations on a $24 \times 32 \times 32$ lattice show that with such a larger transverse size, the overlaps decrease by a factor $\sim 5-10$. Thus we conclude that the overlaps for $t \neq 0$ are also very small, and the low-lying eigenstates cannot be comparable mixtures of $k=2 A$ and $k=2 S$. To make this more precise we shall now turn to an analysis of the actual eigenstates.

\section{$5.2 \quad$ state overlaps}

What we will do in this Section is to estimate the overlaps of the actual string eigenstates onto the $k=2 A$ and $k=2 S$ sectors. Our approach to this question, which is somewhat different to that employed in [5], is as follows.

Let $\left\{\tilde{\Psi}_{i}\right\}$ be the true energy eigenfunctionals ordered, as usual, by their energy $\left\{E_{i}\right\}$. Using only our $k=2 A$ basis, let the variationally calculated trial eigenfunctionals be $\left\{\tilde{\Phi}_{2 A, i}\right\}$.

Suppose now that $\tilde{\Phi}_{2 A, j}$ corresponds to $\tilde{\Psi}_{i}$, where $j \leq i$. (The possibility $j<i$ arises when there is an eigenstate $\Psi$ with a small enough overlap onto our $k=2 A$ basis, that it does not lead to a corresponding $\tilde{\Phi}_{2 A}$, so that the set of variational trial eigenfunctions is incomplete.) Then the normalised correlation function of $\tilde{\Phi}_{2 A, j}$ will be $\simeq \gamma_{2 A}^{2} \exp \left\{-E_{i} t\right\}$ in some range of $t$ - the effective energy plateau. Then $\gamma_{2 A}$ gives us the overlap of the eigenstate $\tilde{\Psi}_{i}$ on $\tilde{\Phi}_{2 A, j}$ and hence, to a good approximation, on our $k=2 A$ basis. Doing the same with the $k=2 S$ basis should give us the corresponding overlap $\gamma_{2 S}$. Now returning to reality, we know that we will not see such a state at all in any sector where its overlap is small. So in practice we can usually only calculate either $\gamma_{2 A}$ or $\gamma_{2 S}$ this way, but not both. So suppose it is $\gamma_{2 A}$ that is large enough to be clearly calculable. Then instead of looking within the $k=2 S$ basis to estimate $\gamma_{2 S}$, we look at the joint basis $\{k=2 A\} \bigoplus\{k=2 S\}$. Here the overlap is $\gamma_{2 A S}$ and must be larger than $\gamma_{2 A}$, so the state will certainly be visible to the variational calculation. If we take the $k=2 A$ and $k=2 S$ bases to be approximately orthogonal to each other, which we have shown above to be the case, then we can estimate $\gamma_{2 S}^{2} \simeq \gamma_{2 A S}^{2}-\gamma_{2 A}^{2}$. If the state is more $k=2 S$ than $k=2 A$ then we follow the same procedure but with $2 A$ with $2 S$ interchanged. So 
in this way we obtain an estimate of $\gamma_{2 A}^{2}$ and $\gamma_{2 S}^{2}$ for each energy eigenstate. These estimates are of course approximate. In particular, our $k=2 A$ and $k=2 S$ bases are not complete, so that $\gamma_{2 A}^{2}+\gamma_{2 A}^{2}<1$ and becomes more so (typically) for higher excited states. In addition the non-orthogonality of the $k=2 S$ and $k=2 A$ sectors introduces an ambiguity in interpreting the results, but this is very small.

We show a selection of such analyses in Fig. 11]. The examples are all from SU(4) at $\beta=50$. (The $\mathrm{SU}(5)$ results would be very similar.) We show results for the $P=+, q=0$ spectrum for the two string lengths shown, as well as results for the longer string length for the $P=-, q=0$ and $P=-, q=1$ sectors. For each state we show our estimate of the $k=2 A$ (red circle) and $k=2 S$ (blue square) overlap squared. We see that all the states are predominantly in one sector or the other. In some cases the sum of the overlaps is significantly less than unity, and that simply reflects the overlap of that state on our whole basis. This effect becomes stronger for the higher excited states.

\section{$6 \omega=2$ 'unbound' fundamental flux loops}

So far we have focused on $k=2$ bound states. However the $k=2$ sector should also include states that consist of a fundamental flux loop that winds twice around the spatial torus, and whose spectrum will be given, to some first approximation, by eqn(13) with $\omega=2$. These states can be thought of as corresponding to two unbound fundamental flux-tubes. Such states might suffer large corrections because in $D=2+1$ such a double winding loop will necessarily cross itself and we know that there are interactions between loops that are strong enough to bind them into the $k=2 A$ strings. Moreover the $\omega=2$ loops should typically be more massive than the corresponding $k=2 A$ states and so they will typically appear in a part of the $k=2$ spectrum where they might be confused with other excited states. All this should make them non-trivial to observe and so what we shall attempt in this Section is no more than a preliminary search for such $\omega=2$ flux loops.

For this purpose we include in our basis additional operators of the form in eqn(4). We then perform the same calculation as in the Section 4 but within this extended basis. Comparing the resulting spectra to those previously obtained in Section 4 , we attempt to identify new states. The new basis of operators that has been used in our calculation consists of approximately $\sim 240$ operators.

In Figs.12 and 13 we compare the $S U(4), P=-$, and $q=0$ spectra obtained with and without the extra $\omega=2$ operators, and we do indeed observe that the former appears to contain two extra states, which we have shown as solid black symbols. For comparison, the blue line represents the NG prediction for a $\omega=2 k=1$ string, using $\sigma=\sigma_{f}$ and $N_{R}=N_{L}=2$ in eqn(13). The red line represents the NG prediction for the $\omega=1 k=2$ antisymmetric representation with the same values of $N_{L}$ and $N_{R}$ and with $\sigma=\sigma_{2 A}$. As we see from Table 1, the ground state of a winding string with $P=-$ and $q=0$ should be two-fold degenerate, and previous calculations for $\omega=1 k=1$ flux tubes [2] have shown that what one gets is one state which is close to the theoretical NG prediction and another one that approaches the theoretical prediction from above as $l$ increases. We observe precisely such a behavior in 
Figure 12, with the lighter state, represented by a star, being close to the $w=2$ prediction and the heavier state, represented by a circle, approaching the blue line from above.

To confirm that these two states are primarily associated with the extra $\omega=2$ operators in our extended basis, we show in Figure 14 the projection in of the variational eigenstates corrsponding to the ground and first excited states onto all the operators in our basis. We have chosen to look at $l=16 a\left(l \sqrt{\sigma_{f}} \sim 2.1\right)$ where these states are (supposedly) the lightest $\omega=1 k=2 A$ and $\omega=2 k=1$ states. For this lattice size we have 160 operators in total, of which the first 100 are our ordinary $k=2$ operators, and the last 60 are our extra $\omega=2$ operators. As can be seen, the ground state projects mainly onto ordinary $k=2$ operators, while the first excited state projects almost entirely onto the $w=2$ sector of the operator basis. This supports our earlier interpretation of these states.

It is interesting to note that the $w=2$ operator that contributes the most to the first excited state is the rectangular pulse (Table 4 , number 3) at blocking level 4 and, therefore, the transverse deformation has a physical length of 16 lattice spacings. A pictorial representation of this operator is included on the panel of Figure 14. We see that it is shaped like an oscillating closed string with two nodes, doubly wound around the torus. This provides further heuristic support for our conclusion that the complete $k=2$ string spectrum includes unbound wavelike $\omega=2$ states.

\section{Looking for extra massive states}

As we remarked in Section 4.1, the interpretation of the first excited state in the $k=2 A$, $P=+, q=0$ spectrum shown in Fig. 6, is ambiguous. It might be the first string excitation, with a large correction to the Nambu-Goto free string energy, which is shown in Fig. 66 and which it appears to be approaching as $l \uparrow$. Alternatively it might be a new excitation that arises from one of the massive modes of the ground state flux tube being excited. Indeed, as we see from Fig. 6, the energy of this state does differ from that of the ground state by roughly a constant as $l$ varies. In this scenario, the energy approaches the energy of the first excited Nambu-Goto state, only to cross it at some larger value of $l$ and in that case it is presumably the next state up in energy which is the true stringy excitation (and which indeed is much closer to the Nambu-Goto value for most of our values of $l$ ).

Since the identification of such extra states would (begin to) provide a completely new source of useful information about the string description of the gauge theory, resolving this ambiguity is important, and that is what we turn to now.

If the state is indeed an approximate Nambu-Goto-like string excitation of the $k=2 \mathrm{~A}$ flux tube, then we would expect its wave-functional to have the appropriate 'shape'. What that 'shape' should be, in terms of our highly blocked/smeared link matrices, is not at all evident, but it is something we do not need to know. It is enough to note that we have already established [2] that the relevant part of the fundamental $k=1$ flux tube spectrum is Nambu-Goto-like. Indeed the $k=1$ analogue of Fig. 6] shows only small corrections to the free string energies. So all we need to do is to compare the wavefunctionals of the first excited $k=1$ and $k=2 A$ states, in exactly the same lattice calculation, and see if they are very 
similar or very different.

The way we make this comparison is as follows. Let $\left\{\phi_{i} ; i=1, \ldots, n_{o}\right\}$ be our set of winding operators where the number is typically $n_{o} \sim 100$. In the present case, we choose them to have $P=+$ and $q=0$. These operators are group elements (not yet traced) and may be in any representation of $\mathrm{SU}(N)$. Suppose the flux is in the representation $\mathcal{R}$. When we perform our variational calculation over this basis, we obtain a set of wavefunctionals, $\Phi_{\mathcal{R}}^{n}$, which are an approximation to the corresponding eigenfunctions of the Hamiltonian. These wavefunctionals are linear combinations of our basis operators:

$$
\Phi_{\mathcal{R}}^{n}=\sum_{i}^{n_{o}} b_{\mathcal{R}, i}^{n} c_{\mathcal{R}, i} \operatorname{Tr}_{\mathcal{R}}\left(\phi_{i}\right) \equiv \sum_{i}^{n_{o}} b_{\mathcal{R}, i}^{n} \operatorname{Tr}_{\mathcal{R}}^{\prime}\left(\phi_{i}\right)
$$

where the coefficients $c_{\mathcal{R}, i}$ are chosen so that we have the normalisation condition

$$
\left\langle\operatorname{Tr}_{\mathcal{R}}^{\prime \dagger}\left(\phi_{i}(0)\right) \operatorname{Tr}_{\mathcal{R}}^{\prime}\left(\phi_{i}(0)\right)\right\rangle=1
$$

The purpose of this common normalisation is to make a comparison of the coefficients $b_{\mathcal{R}, i}^{n}$ for different $\mathcal{R}$, meaningful.

The basic idea now is that the coefficients $b_{\mathcal{R}, i}^{n}$ encode the 'shape' of the state corresponding to the wavefunctional, because they multiply the same operators, albeit in different representations, and with a common normalisation. So to compare our excited $k=1$ and $k=2 A$ wavefunctionals we need simply compare their coefficients $b_{f, i}^{n}$ and $b_{2 A, i}^{n}$. This would be straightforward if the $\phi_{i}$ basis operators were orthogonal, but since they are not, and some have very large overlaps with each other, apparently different sets of $b_{i}$ 's can in fact correspond to very similar wavefunctionals. Normally the obvious way to surmount such obstacle would be to calculate directly the overlap of the two wavefunctionals we want to compare. Unfortunately here that does not work because the operators have different $\mathrm{N}$-ality so that the overlap will be zero. So to proceed we need to transform the $N$-ality of the $k=2 \mathrm{~A}$ wavefunctional to $k=1$ while preserving its essential spatial characteristics. We do so by the simple substitution (with $t=0$ throughout)

$$
\Phi_{2 A}^{n}=\sum_{i}^{n_{o}} b_{2 A, i}^{n} \operatorname{Tr}_{2 A}^{\prime}\left(\phi_{i}\right) \quad \longrightarrow \quad \tilde{\Phi}_{2 A}^{n}=\sum_{i}^{n_{o}} b_{2 A, i}^{n} \operatorname{Tr}_{f}^{\prime}\left(\phi_{i}\right)
$$

where we replace $\operatorname{Tr}_{2 A}^{\prime}$ by $\operatorname{Tr}_{f}^{\prime}$ in the expression for $\Phi_{2 A}^{n}$. Intuitively it is plausible to think of $\tilde{\Phi}_{2 A}^{n}$ as having approximately the same 'shape' as $\Phi_{2 A}^{n}$, so that if we now compare $\tilde{\Phi}_{2 A}^{n}$ directly with any $\Phi_{f}^{n^{\prime}}$ by the calculation of their normalised overlap

$$
O_{n, n}=\frac{<\Phi^{n^{\prime} \dagger} \tilde{\Phi}_{2 A}^{n}>}{<\Phi^{n^{\prime} \dagger}{ }_{f} \Phi_{f}^{n^{\prime}}>^{1 / 2}<\tilde{\Phi}_{2 A}^{n \dagger} \tilde{\Phi}_{2 A}^{n}>^{1 / 2}}
$$

we are in fact comparing the shape of $\Phi_{f}^{n^{\prime}}$ with that of our original $\Phi_{2 A}^{n}$ wavefunctional.

The above method of comparison clearly has a strong heuristic component and to some extent our confidence in the result will depend on how clear-cut it proves to be. So we take our 
four lightest $k=2 A$ (variational) eigenstates, form the corresponding set of $\left\{\tilde{\Phi}_{2 A}^{n} ; n=0, \ldots, 3\right\}$, and take the overlap of each one of these with the corresponding $k=1$ (variational) eigenstates, $\left\{\Phi_{f}^{n} ; n=0, \ldots, 3\right\}$. Note that the former are not necessarily orthogonal, while the latter are, by construction (just as were the original $\left\{\Phi_{2 A}^{n}\right\}$ ). We begin by showing the results, in Fig. 15, for our longest $l=32 a$ flux tube in $\mathrm{SU}(5)$. We see that the $k=2 A$ ground state has an overlap that is virtually $100 \%$ on the $k=1$ ground state. Since we have already established that both ground states are to a very good approximation described by the free string theory, this result serves to give us confidence in this method of comparison. Turning now to the first excited $k=2 A$ state, which is the one whose identity we are most interested in here, we see from Fig. 15 that this has an overlap that is almost entirely on the first excited $k=1$ state. Since we have established that the latter is a Nambu-Goto-like stringy excitation, we infer that so is the first excited $k=2 A$ state. Thus we have our answer: this state is tending to Nambu-Goto and is not an 'extra' state involving massive excitations of the flux tube.

We also see from Fig. 15 that the next two $P=+, q=0 k=2 A$ excited states are very similar to the corresponding $k=1$ states and therefore also Nambu-Goto-like. In Fig. 16 we show the corresponding plots for length $l=16 a$. The results for the ground and first excited states are unambiguous, but the comparison for the higher excited states is now less clear-cut. Perhaps not surprisingly, the states become more clearly Nambu-Goto-like as we go to longer strings.

We have repeated this whole exercise for the $k=2 S$ representation. Once again we find that the ground state has an almost $100 \%$ overlap onto the $k=1$ ground state. However the first excited state is less clear-cut than for $k=2 A$, and the comparison for other states produces no useful information at all.

We have learned that the first excited $k=2 A$ state in the $P=+, q=0$ sector is in fact Nambu-Goto-like, so it has two phonons of momentum $p= \pm 2 \pi / l$. The fact that there is such a large correction to the free string energy when $l$ is not very large, tells us that these phonons have a large interaction energy. On the other hand we have seen that the ground state $k=2 A$ state with $P=+, q=2$ has an energy very close to the free string energy at all $l$. This state has two phonons of the same momentum $p=2 \pi / l$. Thus we infer that the interaction energy of two phonons with the same momentum is very small. So although we have not found an 'extra' state, we have learned something very specific and interesting about the inter-phonon interactions.

\section{Conclusions}

In this paper we have examined to what extent $k=2$ flux tubes that are wound around a spatial torus can be described by an effective string theory. This study complements our earlier study of fundamental $(k=1)$ flux loops [2], where we found that a simple Nambu-Goto free string description described the spectrum very well, even when the length of the flux tube was on the order of its width. Since one can regard a $k=2$ flux loop as a bound state of two $k=1$ flux loops, one would naively expect the extra internal structure to lead to larger corrections to any asymptotic free string description, and indeed that there should be extra 
states that do not look like excited string states and which arise through the excitation of the massive binding modes.

Because $k=2$ states are much more massive than $k=1$ states, some systematic errors are much more important for the former. This has meant that our most reliable calculations are for the ground states in the various sectors labeled by different quantum numbers. These quantum numbers are the parity $P$ and the momentum along the flux tube, $q$. Since we expect the ground state unexcited flux loop to be invariant under translations along its axis, and to be symmetric under reflections across its axis, having $P \neq+$ and/or $q \neq 0$ explicitly probes the non-trivial excitation spectrum of the flux loop (in a way that transverse momenta do not, which is why we do not consider them).

In addition to these exact quantum numbers, we have confirmed by explicit calculation that there is very little overlap between the totally symmetric $(k=2 S)$ and totally antisymmetric $(k=2 A)$ representations. We thus find that our variational eigenfunctionals fall into these representations to a good approximation, so that we can categorise the spectra by their behaviour under the full $\mathrm{SU}(N)$ group, and not just its center $Z_{N}$.

In the $k=2 A$ sector we found that the ground states for $q=0,1,2$ have energies that are very close to those of the free bosonic string theory (Nambu-Goto in flat $D=2+1$ ). The leading permitted correction to $(E(q=0 ; l) /(\sigma l))^{2}$ is $O\left(1 /(\sqrt{\sigma} l)^{5}\right)$, and its fitted coefficient has a natural value of $O(1)$. This is in contrast to the fundamental flux loop where the corresponding coefficient is unnaturally tiny, $<O(1 / 10)$. More surprising is that the $q=1,2$ ground state energies are well described by Nambu-Goto all the way down to $l \sqrt{\sigma} \simeq 1.5$. Such a flux tube is a blob rather than a string and it is remarkable that the thin string oscillation spectrum should accurately describe its lowest modes. Just as for the $k=1$ flux tube, one is tempted to read into this evidence for some kind of gauge-string duality that makes a thin string the starting point for describing even a short blob-like flux tube. For the low-lying excited states in the $q=0$ sector the deviations are much larger - and of a size that one would expect within an effective string theory description that was only valid asymptotically.

The much heavier $k=2 S$ sector is clearly afflicted by much larger systematic errors, which strongly limits our ability to draw any useful conclusions, although here too the ground states of various momenta $q$ are recognisably string like.

As an interesting aside, by including operators that wound twice around the torus, but such that the second winding overlapped little in space with the first, we were able to identify some eigenstates that correspond to doubly wound fundamental flux loops, in addition to the bound $k=2$ singly wound flux loops.

While it is very informative to observe how well the various states are described by simple free string excitations, this can only be the first step. We also need to pin-point the nature of the corrections to the free string theory. This involves not only quantifying and searching for patterns in the corrections to the free string excitations - the interactions amongst the phonons - but also identifying excited states that are additional to those associated with simple string oscillations. Such additional states, due to excitations of massive modes, would normally be predicted by any theoretical approach.

For example, in a naive field theoretic approach, where the flux tubes are some kind of dynamically generated non-Abelian Nielsen-Olesen vortices, there should be massive excitations 
associated with the nontrivial flux tube structure. In an 'AdS/CFT' approach there would be a highly curved metric in the region of the higher dimensional space where the string picks up its non-trivial energy per unit length. Naively, the energy of such an 'extra' state should be some (roughly) constant amount $\mu$ above the ground state energy. As we remarked earlier, a naive estimate for $\mu$ might be the lightest glueball mass, $\sim 4 \sqrt{ } \sigma_{f}$, or possibly the gap between the lightest glueball masses, $\sim 2 \sqrt{ } \sigma_{f}$. On such an estimate, such states should be easily visible for small flux tube lengths, where they would be lighter than some of the simple thin string excitations. A glance at the $q=0$ spectra in Figs. [5] and 6, immediately suggests that the first excited state could be a candidate for such a state. However our analysis in Section 7 of the wavefunctional of that state showed that its 'shape' was too much like that of the corresponding $k=1$ flux tube, which is unambiguously Nambu-Goto-like, to believe that it is anything other than a simple string excited state. We have in fact not observed any states, in the range of energies where we have some control, that could be interpreted as such non-stringy excitations. This could either mean that we have a poor overlap onto such states or that their masses are much larger than expected. (The glueball mass might not be a good guide if its dynamics is that of a string-like contractible closed loop of flux, so that neither its mass nor the gap to the first excited state are strongly influenced by the dynamics associated with the non-stringy massive modes.) In the former case it means that we must suitably extend our basis of operators; and for that purpose it would be useful to have some guidance from, say, the gauge-gravity side, about the lightest such extra states. In the latter case, the implication would be that the non-stringy physics was essentially decoupled from the low-energy physics of the confining gauge theory. Resolving this issue would provide something very interesting in either case.

Although the question of non-stringy 'extra' states has not been settled in this paper, we have been able to identify some qualitative features of the interactions amongst the phonons along the flux tube. As we have just remarked, we have shown that the first excited $q=0$ $P=+$ state is in fact a string excited state, i.e. it has two phonons of opposite momentum $p= \pm 2 \pi / l$. And, as we see in Figs. 5 and 6, the energy of this state has very large corrections to the corresponding free string energy. On the other hand the lightest $q=2 P=+$ state, which has exactly the same two phonons, but with the same momentum, has as we see in Figs. 1 and 2, an energy that is virtually identical to that of the corresponding free string state, over our whole range of $l$. Thus we can come to a very specific conclusion about the nontrivial interactions between two phonons: they are negligible when the phonons have exactly the same momenta (and are therefore at 'threshold'), but are large when the momenta are exactly opposite.

One might ask why such a conclusion had not already been reached in our much more accurate calculations of the fundamental $k=1$ flux loop spectrum in [2]. There are at least two plausible reasons. Firstly, we have seen that the corrections are in general much smaller for $k=1$ than for $k=2$ flux loops, so much so that the first excited $q=0 P=+k=1$ flux loop looks, at a first glance, to be close to the free string prediction. Secondly, we observed in [2] some significant corrections in the $q=2$ spectrum at small $l$, in contrast to the case of $k=2$. However we have since been able to show that these are largely due to lattice spacing corrections to the energy-momentum dispersion relation. Thus a renewed analysis 
may well show that the $k=1$ flux loop displays the same kind of non-trivial interaction between phonons that we have seen with the $k=2$ loop. The fact that the $k=2$ calculation in this paper has been performed at a smaller value of $a$, together with the fact that the $k=2$ loops are much heavier and therefore less sensitive to the momentum and to any lattice corrections thereof, may be part of the reason that it has been easier to identify this effect in the present, nominally more difficult, calculation.

There is of course much more information about the phonon interactions, that is implicit in our calculations. We have focused on the above single result because it involves energy levels that are non-degenerate, making the argument and conclusion particularly straightforward. In most other cases corrections to the Nambu-Goto energy levels are accompanied by splittings and (presumably) mixing of the would-be degenerate string states, and this will complicate the analysis. While there are other examples of non-degenerate states that can be usefully compared, e.g. the ground state in the $P=+, q=3$ sector and the first excited state in the $P=+, q=1$ sector, these involves values of momentum $q>2$ which have not been studied in the $k=2$ calculation of the present paper (but have been included in our accompanying $k=1$ calculations). We therefore leave a more complete analysis to a forthcoming publication [17] that will describe in detail all our $k=1$ and $k=2$ calculations.

\section{Acknowledgements}

MT thanks the Galileo Galilei Institute for its hospitality during the Workshop on NonPerturbative Methods in Strongly Coupled Gauge Theories and various participants throughout the workshop for very useful and informative discussions. The computations were performed on resources funded by Oxford and EPSRC. AA was supported by the EC $6^{\text {th }}$ Framework Programme Research and Training Network MRTN-CT-2004-503369 and the Leventis Foundation. BB was supported in part by the U.S. Department of Energy under Grant No. DE-FG02-96ER40956. 


\section{Appendix A: Construction of operators}

To calculate the excitation spectrum of $k=2$ strings, it is necessary to find a way to project onto such states. The way to achieve this is to find a suitable basis described by good quantum numbers, in which our operators will be encoded. In our case, this basis is defined by the quantum numbers of momentum, $q$, along the string, and the parity $P$, where the latter tells us how a string transforms under reflections in its transverse axis. This imples that we need to introduce transverse deformations in Polyakov loops and construct line paths that transform in a certain way under such parity reflections and longtitudinal momentum. In general, the more operators we use the better the results we obtain; we therefore construct a plethora of Polyakov paths trying to extract states with high overlaps.

In the first step of the calculation we construct the most commonly used $k=2$ string operators: $\left(\operatorname{Tr}\left\{l_{p}\right\}\right)^{2}$ and $\operatorname{Tr}\left\{l_{p}^{2}\right\}$. Our desire to extract the excitation spectrum and introduce more degrees of freedom, such as the transverse parity and momentum, forces us to modify them and thus to complicate their structure, as discussed in Section 2.3. In Eq. (30-32) we demonstrate how these new operators are composed for the $k=1,2$ cases. For simplicity, below we demonstrate the way our operators have been constructed with a particular transverse deformation that is easy to visualize. First are the negative and positive parity operators for the $k=1$ strings:

$$
\Phi_{k=1}^{P= \pm}=\operatorname{Tr}\{\longleftarrow\} \pm \operatorname{Tr}\{\square-\} .
$$

From these we construct the two simplest sets of positive and negative parity operators for the $k=2$ string, i.e. those corresponding to $\operatorname{Tr}\left\{l_{p}\right\}^{2}$

$$
\Phi_{k=2}^{P= \pm}=\operatorname{Tr}\{\square\}^{2} \pm \operatorname{Tr}\{\square\}^{2},
$$

and those corresponding to $\operatorname{Tr}\left\{l_{p}^{2}\right\}$

$$
\Phi_{k=2}^{P= \pm}=\operatorname{Tr}\{\square \cdot \square\} \pm \operatorname{Tr}\{\square \cdot \square\ulcorner\} .
$$

Next, we project onto the $k=2$ totally antisymmetric and symmetric representations. To single-out the irreducible representations which describe the theory, we perform antisymmetrisation and symmetrisation according to the relevant Young-tableau decomposition of $k=2$ fundamental colour sources: $\square \otimes \square=\square \square \bigoplus \square$. The resulting operators will be of the type: $\operatorname{Tr}\left\{l_{p}\right\}^{2} \pm \operatorname{Tr}\left\{l_{p}^{2}\right\}$, where $+(-)$ for symmetric(antisymmetric) representation. Once more we need to introduce transverse deformations, in order to project onto the non-trivial irreducible representations that characterise the closed flux tube in $D=2+1$. Examples of such operators are demonstrated in Eqs. (33, 34) below. Begining with the projection onto the $k=2$ antisymmetric representation we find

$$
\Phi_{k=2 A}^{P= \pm}=\left[\operatorname{Tr}\{\square\}^{2}-\operatorname{Tr}\{\neg \cdot \square\}\right] \pm\left[\operatorname{Tr}\{\square-\}^{2}-\operatorname{Tr}\{\square \sqsubset \cdot \square-\}\right],
$$

and projecting onto the $k=2$ symmetric representation one obtains

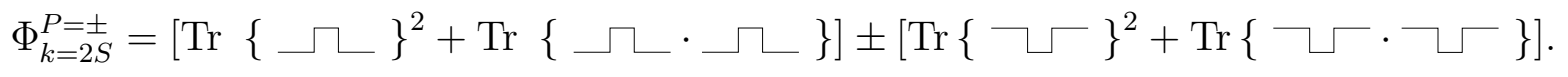


As the notation suggests, in both cases above the \pm signs determine the parity $P= \pm$ of $\Phi$. The complete set of polyakov lines used in our calculation is presented in Table 4 .

As mentioned in Section 2.3, we have also attempted to test whether the $k=2$ string spectrum includes unbound $w=2$ states, which we expect will only appear in our calculation if we use the appropriate operators. These new states are expected to be described by frequencies lower than those describing the $w=2$ bound states. Although some $k=2$ operators look as if they wind twice around the torus i.e Eqs. (31] 32), the way we had been constructing them prohibits us to project onto states with lower frequencies since each Polyakov loop starts and ends at the same lattice point within one lattice size. To overcome this we construct Polyakov lines that wind twice around the torus with transverse deformations at the joint of the two lattices as in :

$$
\Phi_{w=2}^{P= \pm}=\operatorname{Tr}\{\square \square+\operatorname{Tr}\{\square \square\}
$$

Note the number of lattice links in the $x$ directions that goes into each of the operators in the traces above is $2 L_{x}$, i.e. twice the lattice size. This is in contrast to the case of Eqs. (31)-(32), where it is only $L_{x}$.

Finally, let us note that the new operators in Eq. 35 transform the same under the centre of the group $Z_{N}$ as the other $k=2$ operators i.e $\Phi_{w=2}=\operatorname{Tr}\left(l_{p} l_{p}^{\prime}\right) \rightarrow z^{2} \operatorname{Tr}\left(l_{p} l_{p}^{\prime}\right)$. So these new 'unbound' operators have $N$-ality $k=2$ and will therefore contribute to an extended $k=2$ spectrum. 


\section{References}

[1] B. Bringoltz and M. Teper, Phys. Lett. B645 (2007) 383 [hep-th/0611286].

[2] A. Athenodorou, B. Bringoltz and M. Teper, Phys. Lett. B656 (2007) 132 arXiv:0709.0693.

[3] K. Peeters and M. Zamaklar, arXiv:0708.1502.

D. Mateos, arXiv:0709.1523.

J. Erdmenger, N. Evans, I. Kirsch and E. Threlfall arXiv:0711.4467.

[4] B. Lucini and M.Teper, Phys. Rev. D64 (2001) 105019 [hep-lat/0107007].

[5] B. Bringoltz and M. Teper, Phys. Lett. B663 (2008) 429 arXiv:0802.1490].

[6] M. Teper, Phys. Rev. D59 (1999) 014512 [hep-lat/9804008].

[7] B. Lucini, M. Teper and U. Wenger, HEP 0406 (2004) 012 [hep-lat/0404008].

[8] J. Liddle and M. Teper, arXiv:0803.2128; PoS LAT2005:188,2006 hep-lat/0509082.

K.Holland, M. Pepe and U-J Wiese, JHEP 0802 (2008) 041 [arXiv:0712.1216].

K.Holland, JHEP 0601 (2006) 023 [hep-lat/0509041].

[9] M. Luscher, K. Symanzik and P. Weisz, Nucl. Phys. B173 (1980) 365.

M. Luscher, Nucl. Phys. B180 (1981) 317.

[10] M. Luscher and P. Weisz, JHEP 0407 (2004) 014 [arXiv:hep-th/0406205].

[11] J. Drummond, arXiv:hep-th/0411017; arXiv:hep-th/0608109.

N. Hari Dass and P. Matlock, arXiv:hep-th/0606265, arXiv:hep-th/0611215.

[12] J. Polchinski and A. Strominger, Phys. Rev. Lett. 67 (1991) 1681.

[13] D. Tong, arXiv:hep-th/0809.5060.

[14] P. Olesen, Phys. Lett. 160B (1985) 144.

[15] J. Arvis, Phys. Lett. 127B (1983) 106.

[16] J. Polchinski, String Theory (CUP, 1998).

[17] A. Athenodorou, B. Bringoltz and M. Teper in preparation. 


\begin{tabular}{||c||c||c||c||c|c||}
\hline \hline level & $N_{R}$ & $N_{L}$ & $q$ & $P=+$ & $P=-$ \\
\hline 0 & 0 & 0 & 0 & $|0\rangle$ & $\alpha_{-1}|0\rangle$ \\
\hline 1 & 1 & 0 & 1 & & $\alpha_{-2}|0\rangle$ \\
\hline 2 & 1 & 1 & 0 & $\alpha_{-1} \bar{\alpha}_{-1}|0\rangle$ & $\alpha_{-1} \alpha_{-1} \bar{\alpha}_{-1}|0\rangle$ \\
\hline 3 & 2 & 0 & 2 & $\alpha_{-1} \alpha_{-1}|0\rangle$ & $\alpha_{-2} \bar{\alpha}_{-1}|0\rangle$ \\
\hline 4 & 2 & 1 & 1 & $\alpha_{-1} \alpha_{-1} \bar{\alpha}_{-2}|0\rangle$ \\
\hline 5 & 2 & 2 & 0 & $\alpha_{-2} \bar{\alpha}_{-2}|0\rangle, \alpha_{-1} \alpha_{-1} \bar{\alpha}_{-1} \bar{\alpha}_{-1}|0\rangle$ & $\alpha_{-2} \bar{\alpha}_{-1} \bar{\alpha}_{-1}|0\rangle, \alpha_{-1} \bar{\alpha}_{-1}|0\rangle$ \\
\hline 6 & 3 & 1 & 2 & $\alpha_{-3} \bar{\alpha}_{-1}|0\rangle, \alpha_{-1} \alpha_{-1} \alpha_{-1} \bar{\alpha}_{-1}|0\rangle$ & $\alpha_{-2} \alpha_{-1} \bar{\alpha}$ \\
\hline \hline
\end{tabular}

Table 1: The seven lowest Nambu-Goto energy levels for the $w=1$ closed string. If the number of creation operators is even(odd) then the state has positive(negative) parity.

\begin{tabular}{|c|c||c|c|c||c|c|}
\hline \multicolumn{7}{|c|}{$O_{A S}(t=0)$} \\
\hline$b_{A}$ & $b_{S}$ & $l=l_{y}=16$ & $l=16, l_{y}=20$ & $l=16, l_{y}=48$ & $l=l_{y}=20$ & $l=l_{y}=24$ \\
\hline 1 & 1 & $0.0002(3)$ & $0.0001(13)$ & $-0.0014(13)$ & $-0.0001(3)$ & $-0.0001(4)$ \\
2 & 2 & $-0.0001(3)$ & $0.0020(10)$ & $-0.0010(9)$ & $0.0001(2)$ & $-0.0001(3)$ \\
3 & 3 & $-0.0001(3)$ & $-0.0004(14)$ & $-0.0003(10)$ & $-0.0001(3)$ & $0.0003(4)$ \\
4 & 4 & $0.0025(3)$ & $0.0001(18)$ & $-0.0017(12)$ & $0.0003(4)$ & $-0.0002(4)$ \\
4 & 5 & $0.1822(3)$ & $0.0589(19)$ & $0.0277(10)$ & $0.0142(4)$ & $-0.0027(4)$ \\
5 & 4 & $0.0353(3)$ & $0.0118(16)$ & $0.0007(13)$ & $0.0040(3)$ & $0.0003(3)$ \\
5 & 5 & $0.2806(4)$ & $0.1335(22)$ & $0.0404(13)$ & $0.0472(4)$ & $0.0005(5)$ \\
\hline
\end{tabular}

Table 2: Overlaps of Polyakov loops in the $\mathrm{k}=2 \mathrm{~A}$ and $\mathrm{k}=2 \mathrm{~S}$ representations, with blocking levels $b_{A}$ and $b_{S}$ respectively, at $t=0$ and as defined by eqn(25). For $\mathrm{SU}(4)$ at $\beta=32.0$ on $l \times l_{y} \times 20$ lattices.

\begin{tabular}{|c|c||c|c|c|}
\hline \multicolumn{5}{|c|}{$O_{A S}(t)$} \\
\hline$b_{A}$ & $b_{S}$ & $t=0$ & $t=1$ & $t=2$ \\
\hline 1 & 1 & $0.0001(2)$ & $0.0075(31)$ & $0.0055(186)$ \\
2 & 2 & $-0.0000(3)$ & $0.0000(10)$ & $-0.0010(27)$ \\
3 & 3 & $0.0002(3)$ & $0.0002(6)$ & $-0.0017(14)$ \\
4 & 4 & $0.0008(4)$ & $0.0010(7)$ & $0.0017(12)$ \\
4 & 5 & $0.0098(5)$ & $0.0151(8)$ & $0.0224(13)$ \\
5 & 4 & $0.0043(4)$ & $0.0059(7)$ & $0.0086(13)$ \\
5 & 5 & $0.0292(5)$ & $0.0380(9)$ & $0.0489(14)$ \\
\hline
\end{tabular}

Table 3: Overlaps of Polyakov loops in the $\mathrm{k}=2 \mathrm{~A}$ and $\mathrm{k}=2 \mathrm{~S}$ representations, with blocking levels $b_{A}$ and $b_{S}$ respectively, at $t=0,1,2$ and as defined by eqn(25). For $\operatorname{SU}(5)$ at $\beta=80.0$ on $24^{2} 32$ lattices. 


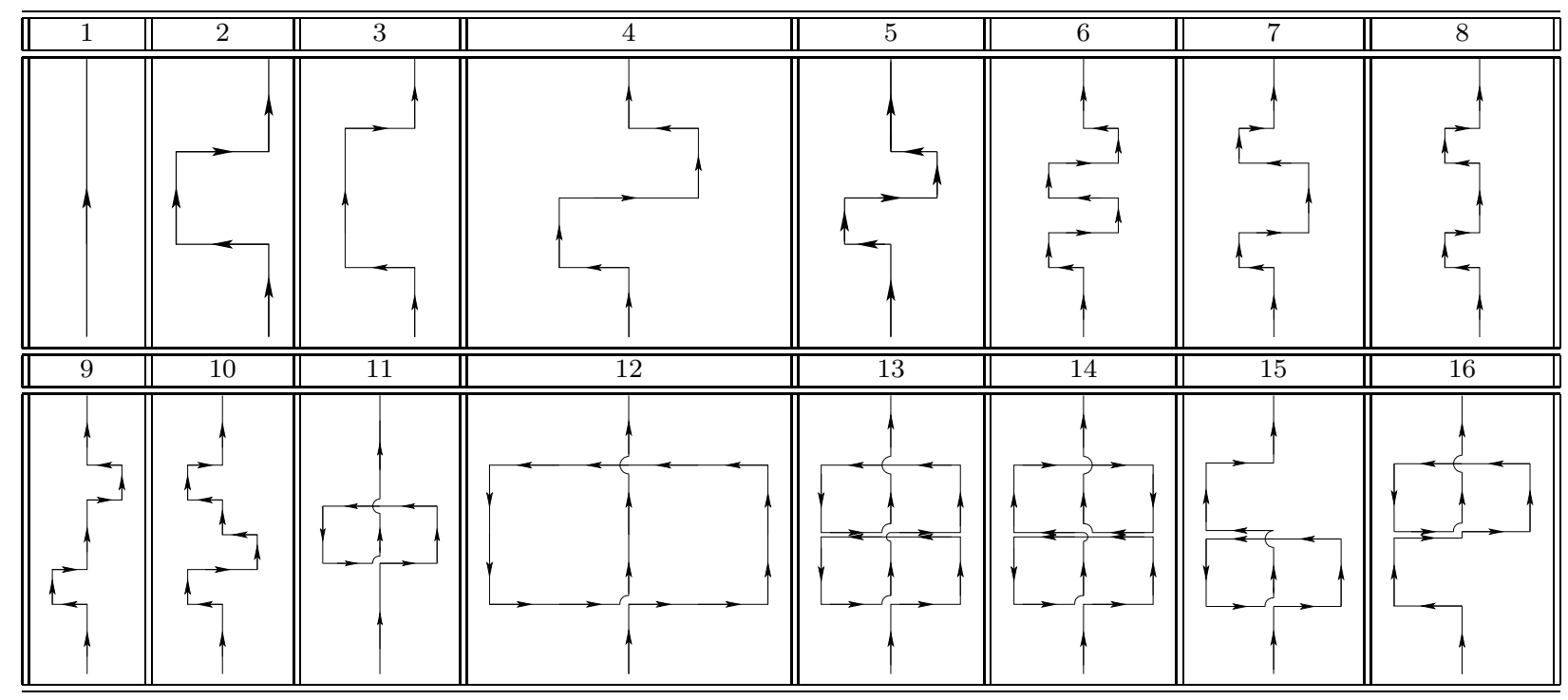

Table 4: The sixteen transverse deformations used in the construction of the operators in this work. Each line comes in five different blocking/smearing levels, in order to enhance the overlap onto the physical states.

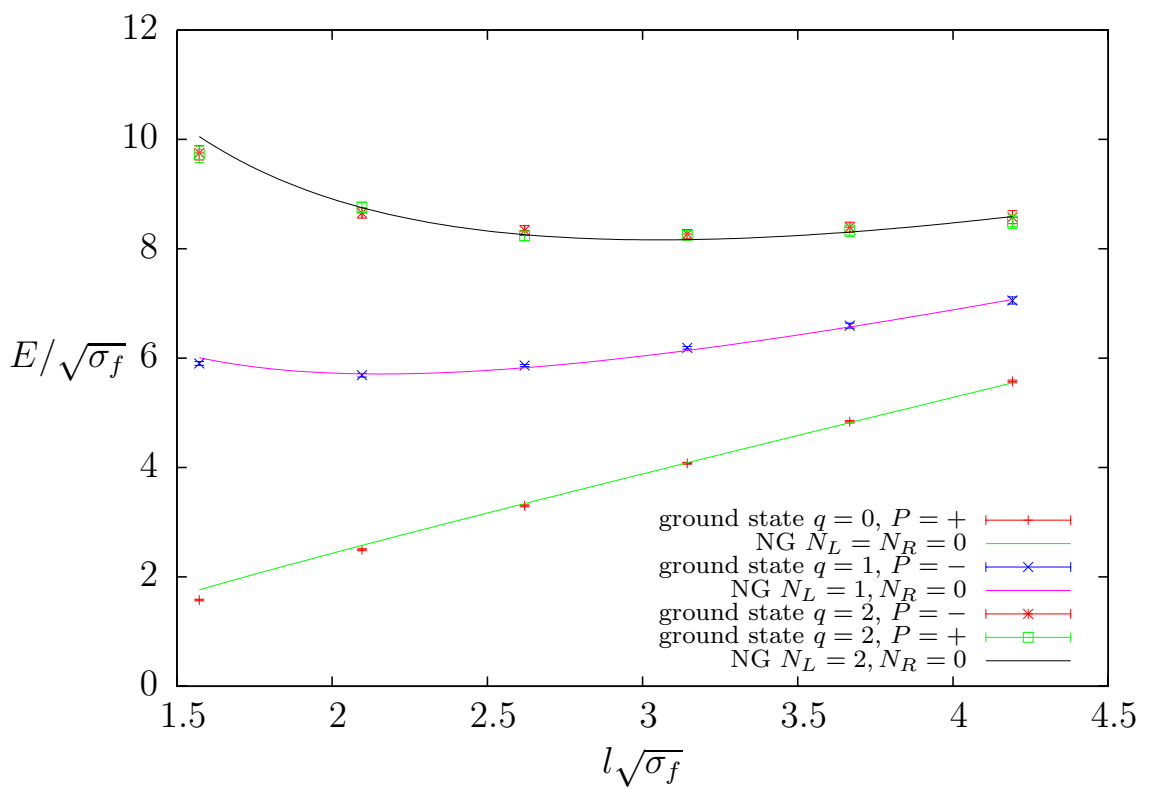

Figure 1: Energies of the lightest four states with $q=0,1,2$, for winding flux tubes in the $k=2$ antisymmetric representation of $S U(4)$ at $\beta=50$. Lines are the Nambu-Goto predictions of eqn(13), with the string tension obtained by fitting the ground state. 


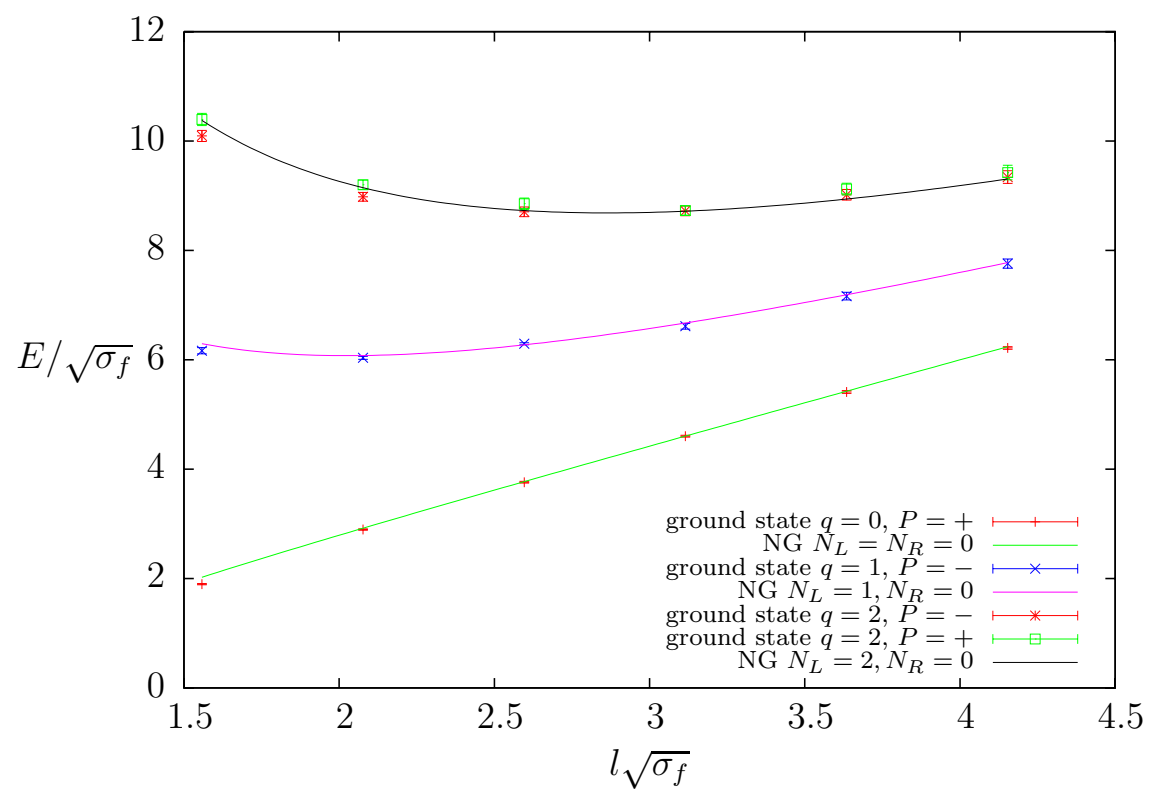

Figure 2: Energies of the lightest four states with $q=0,1,2$, for winding flux tubes in the $k=2$ antisymmetric representation of $S U(5)$ at $\beta=80$. Lines are the Nambu-Goto predictions of eqn(13), with the string tension obtained by fitting the ground state.

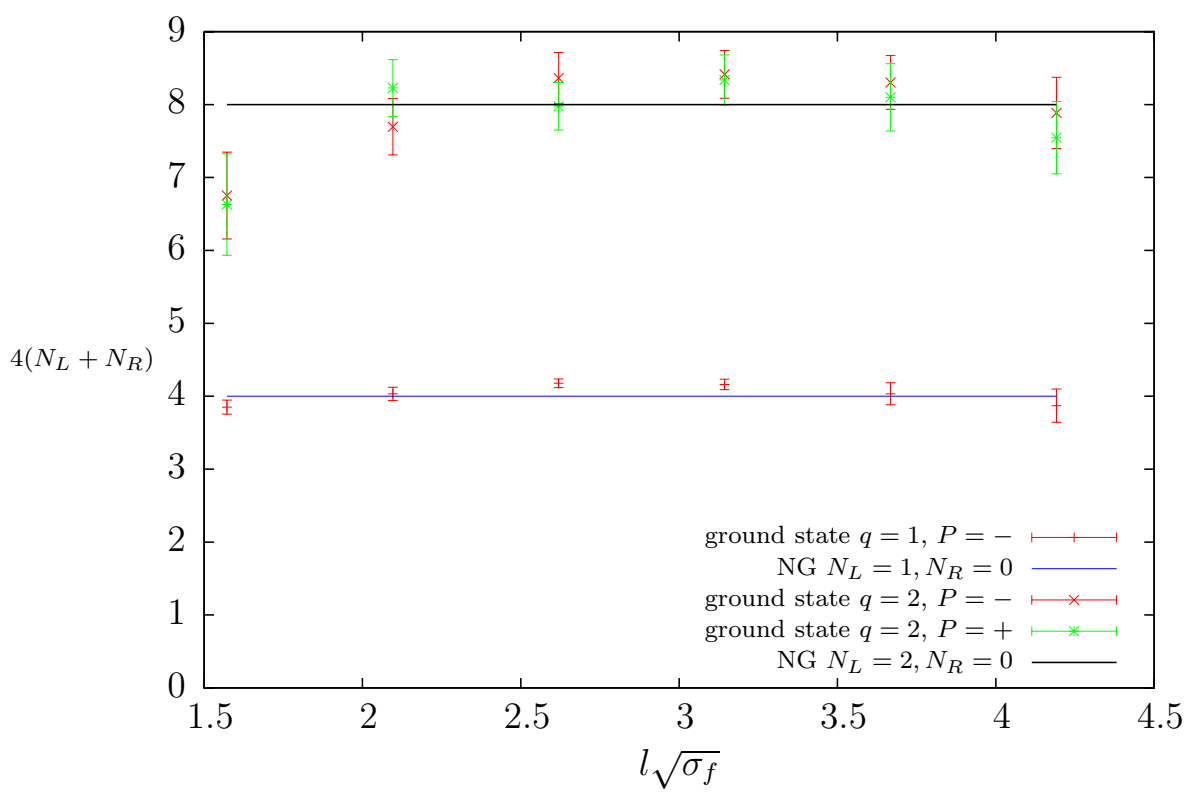

Figure 3: Flux tube excitation energies from the $\mathrm{SU}(4) q=1,2$ calculations in Fig. 1, extracted using eqn(21). Lines are the Nambu-Goto predictions from eqn(13). 


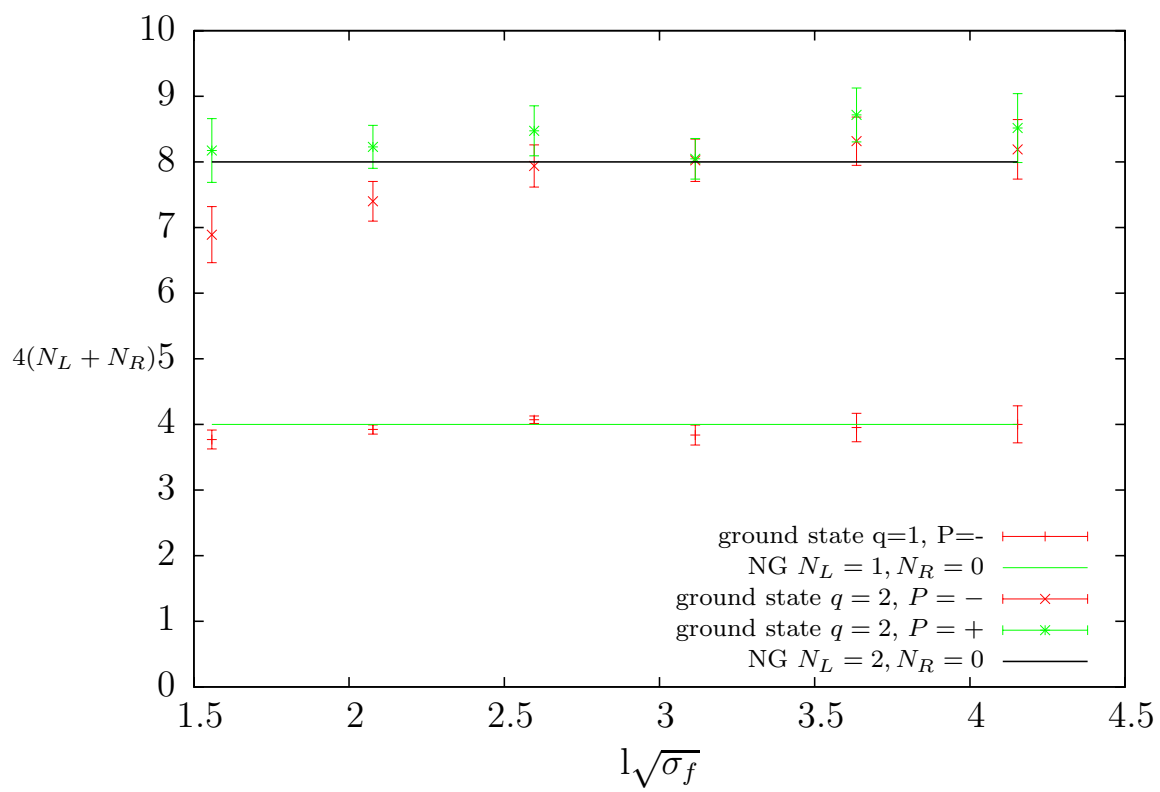

Figure 4: Flux tube excitation energies from the $\mathrm{SU}(5) q=1,2$ calculations in Fig. 2, extracted using eqn(21). Lines are the Nambu-Goto predictions from eqn(13).

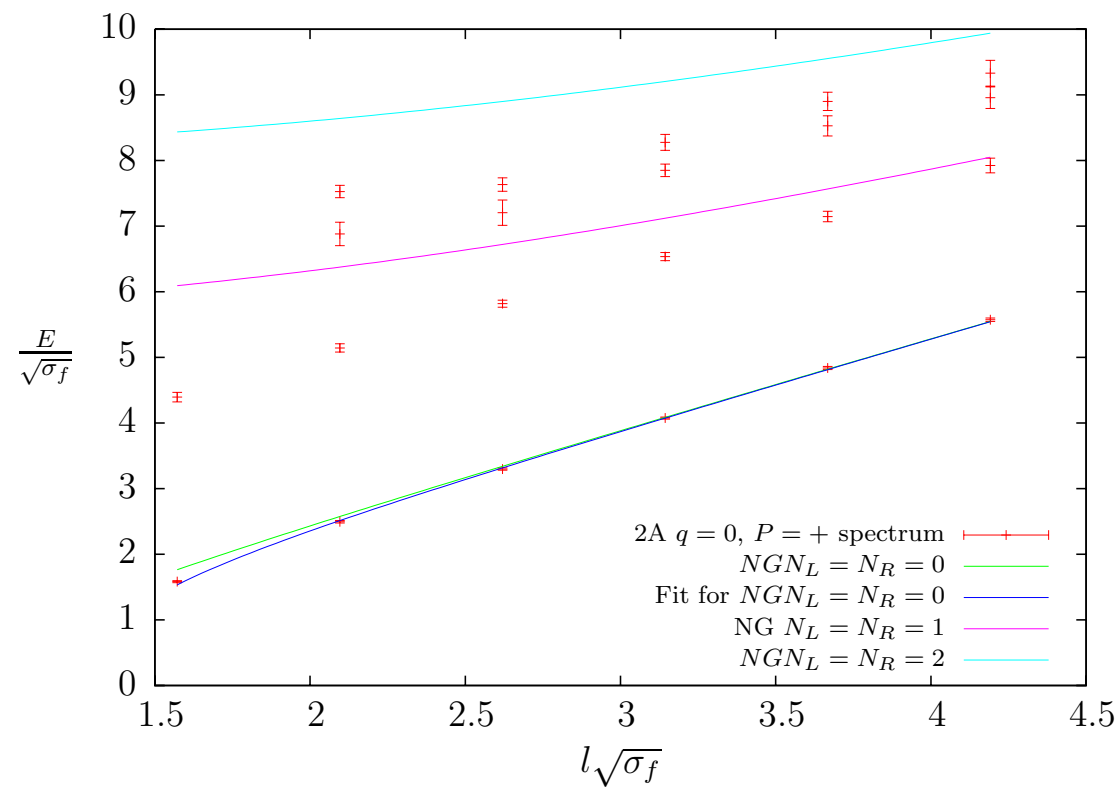

Figure 5: Energies of the lightest four states with $P=+$ and $q=0$, for winding flux tubes in the $k=2$ antisymmetric representation of $S U(4)$ at $\beta=50$. Lines are the Nambu-Goto predictions of eqn(13), with the string tension obtained by fitting the ground state. 


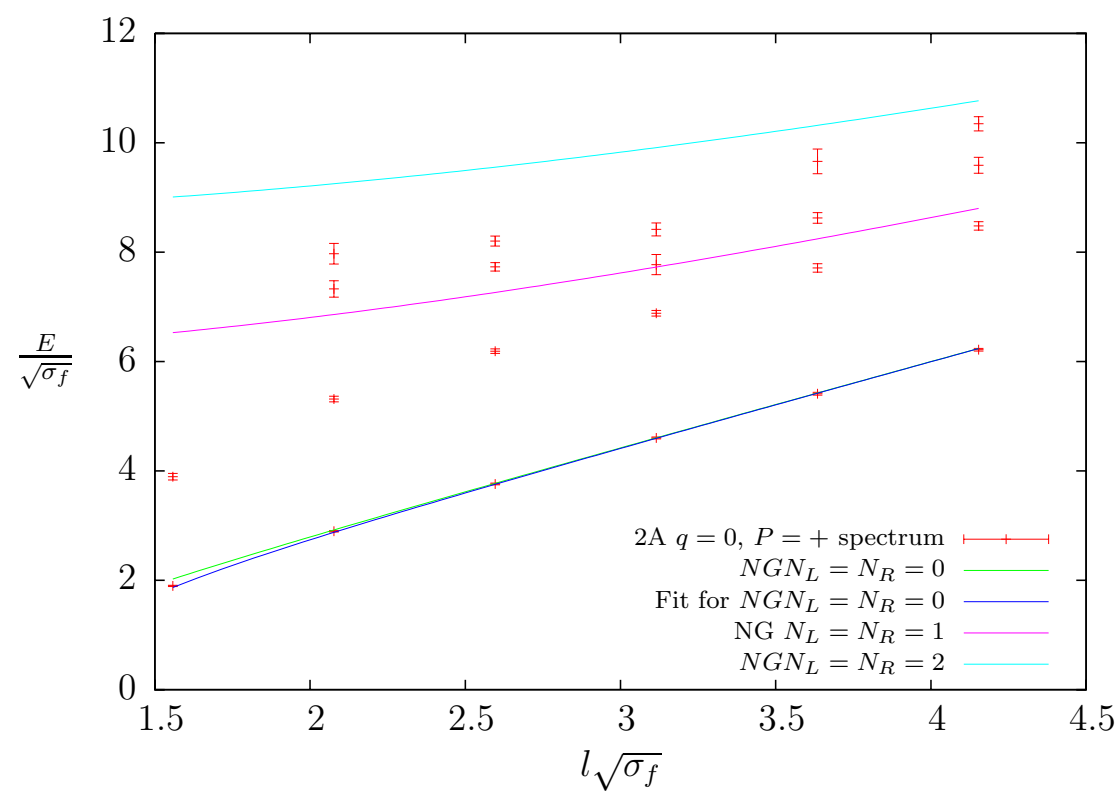

Figure 6: Energies of the lightest four states with $P=+$ and $q=0$, for winding flux tubes in the $k=2$ antisymmetric representation of $S U(5)$ at $\beta=80$. Lines are the Nambu-Goto predictions of eqn(13), with the string tension obtained by fitting the ground state.

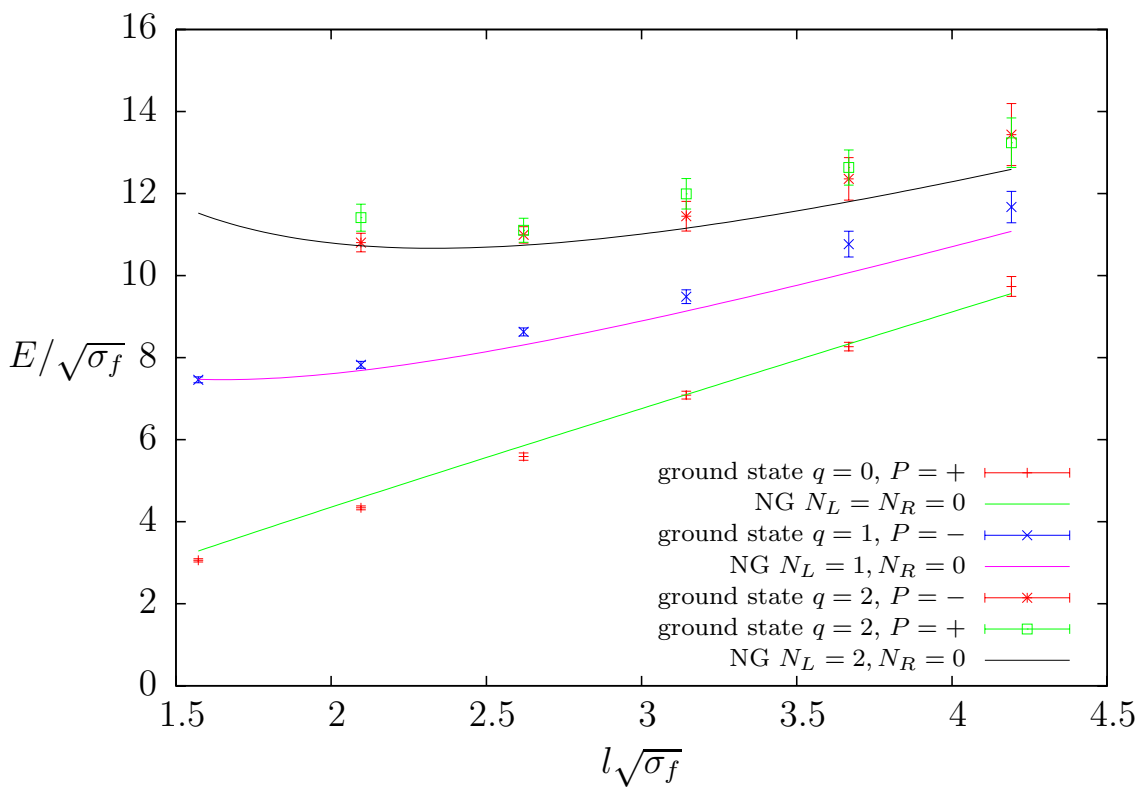

Figure 7: Energies of the lightest four states with $q=0,1,2$, for winding flux tubes in the $k=2$ symmetric representation of $S U(4)$ at $\beta=50$. Lines are the Nambu-Goto predictions of eqn(13), with the string tension obtained by fitting the ground state. 


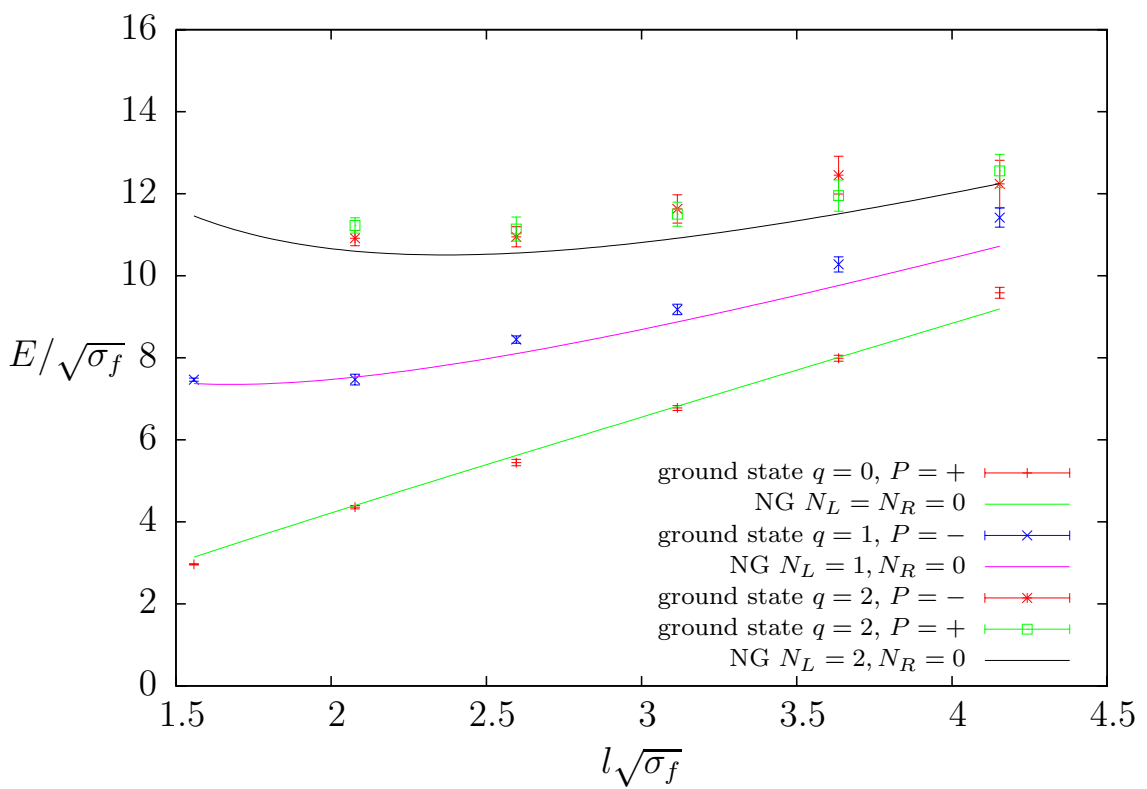

Figure 8: Energies of the lightest four states with $q=0,1,2$, for winding flux tubes in the $k=2$ symmetric representation of $S U(5)$ at $\beta=80$. Lines are the Nambu-Goto predictions of eqn(13), with the string tension obtained by fitting the ground state.

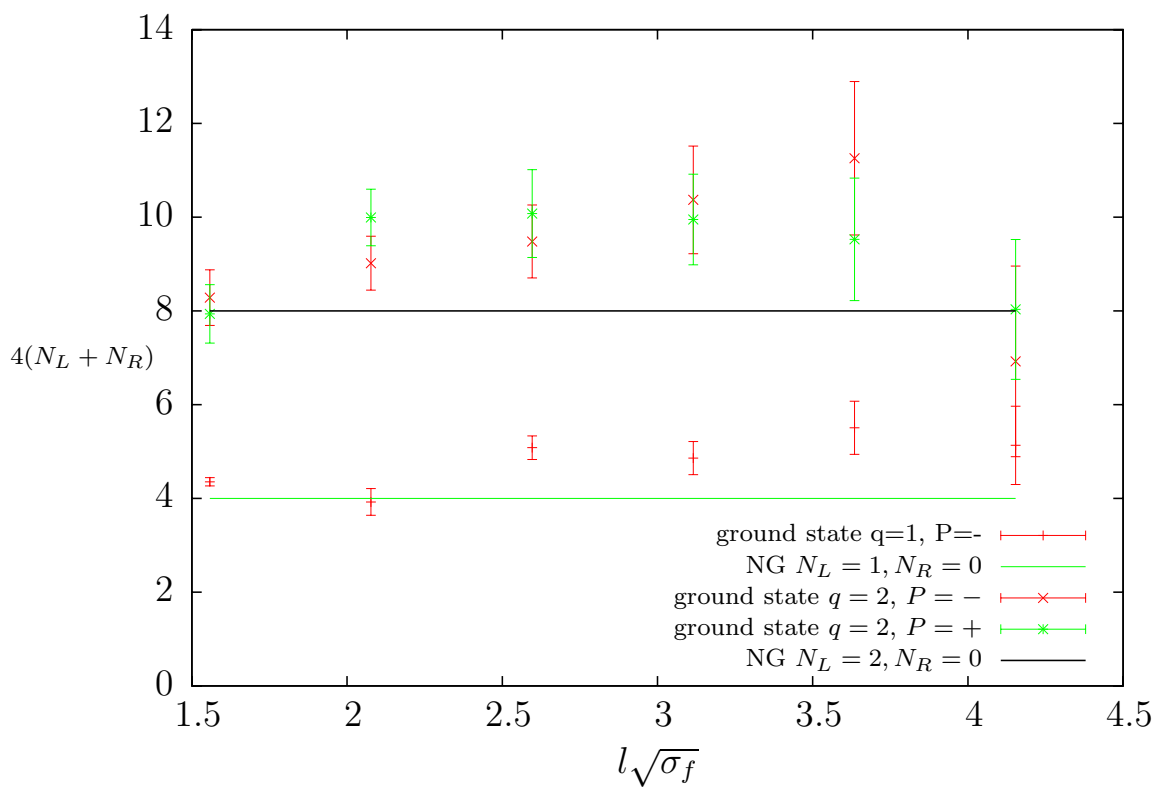

Figure 9: Flux tube excitation energies from the $\mathrm{SU}(5) q=1,2$ calculations in Fig. 8, extracted using eqn(21). Lines are the Nambu-Goto predictions from eqn(13). 


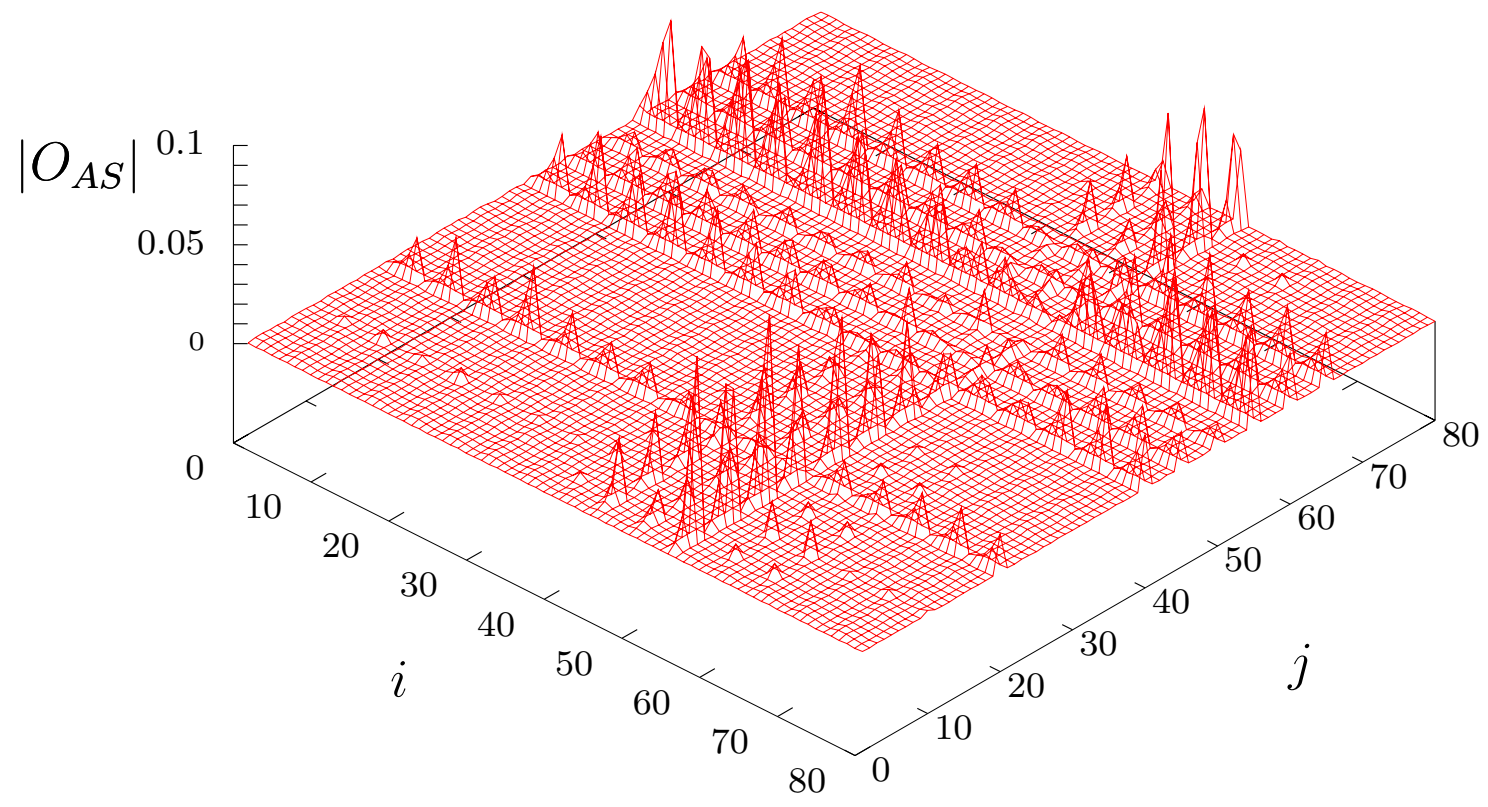

Figure 10: The overlap $\left|O_{A S}(i, j ; t=0)\right|$ as defined by eqn(25) for $S U(4), L=32 a, P=+$ and $q=0$ with $i, j=1-80$. 

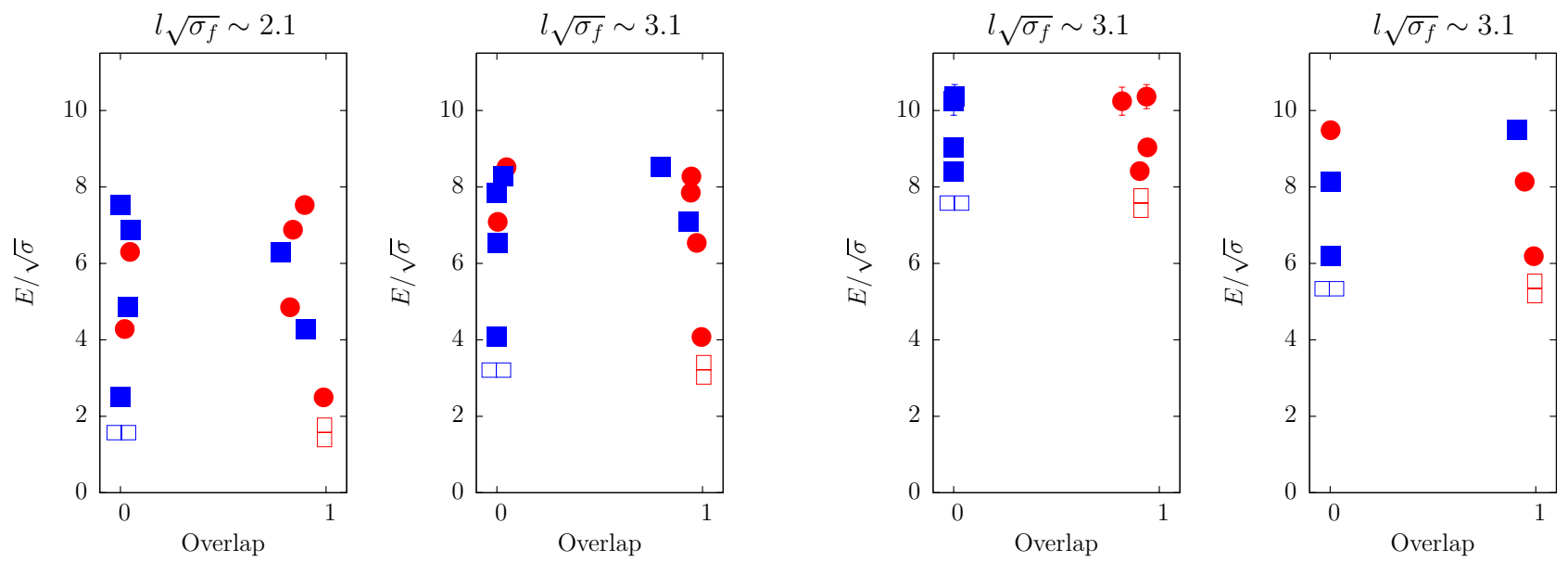

Figure 11: Overlaps squared onto $k=2 A$ (red circle) and $k=2 S$ (blue square) of the low-lying $k=2$ flux tube states with the lengths $l$ shown. In sectors, from left to right, $\{P=+, q=0\}$, $\{P=+, q=0\},\{P=-, q=0\},\{P=-, q=1\}$. All in $\mathrm{SU}(4)$ at $\beta=50$.

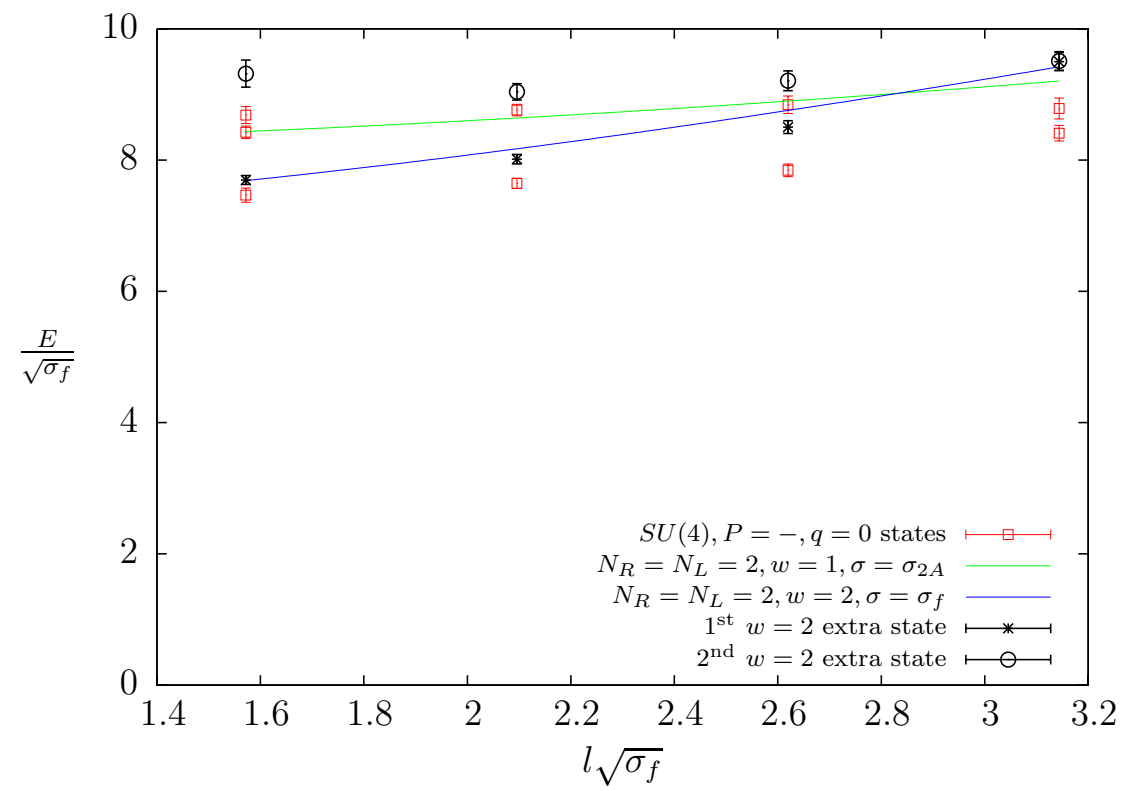

Figure 12: Spectrum of $k=2$ flux loops with $P=-$ and $q=0$, as extracted using the extended basis of operators, that includes $w=2 k=1$ operators as in eqn(4). 


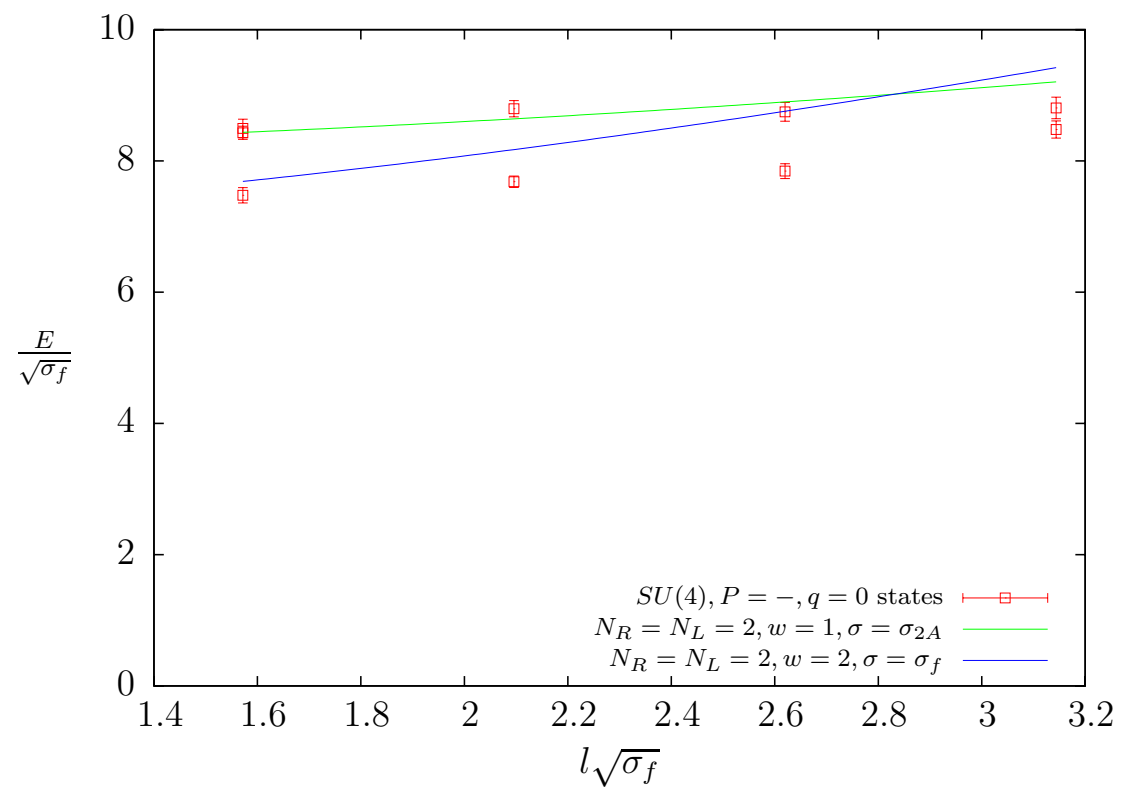

Figure 13: Spectrum of $k=2$ flux loops with $P=-$ and $q=0$, as extracted using only our usual normal $k=2$ basis of $\{k=2 A\} \bigoplus\{k=2 S\}$ operators, as in eqn(3), and excluding the $\omega=2$ states

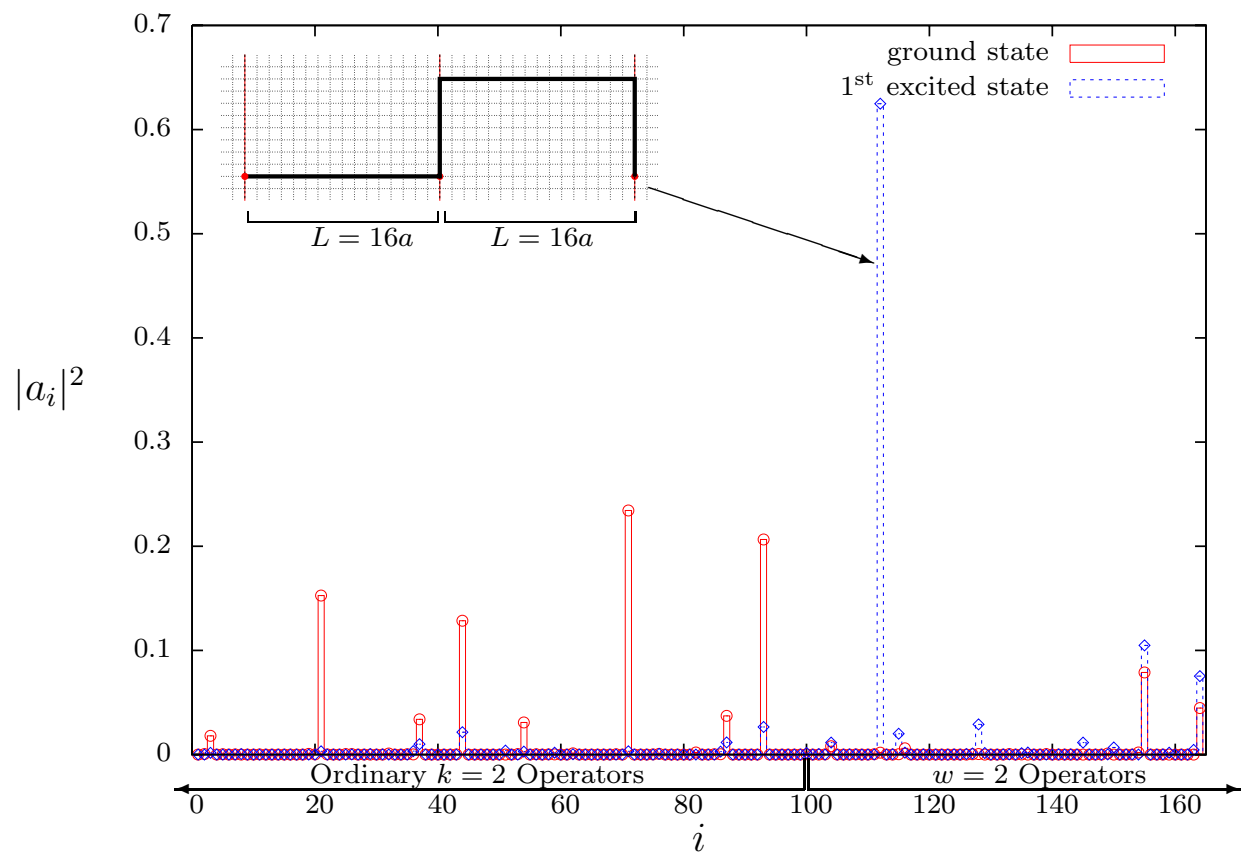

Figure 14: The contribution of each of our extended basis of operators to the ground and first excited states shown in Fig. 12 for $L=16 a$. For $i \leq 100$ the operators are the ordinary $k=2$ ones and for $i>100$ the operators are the additional $w=2$ ones. 


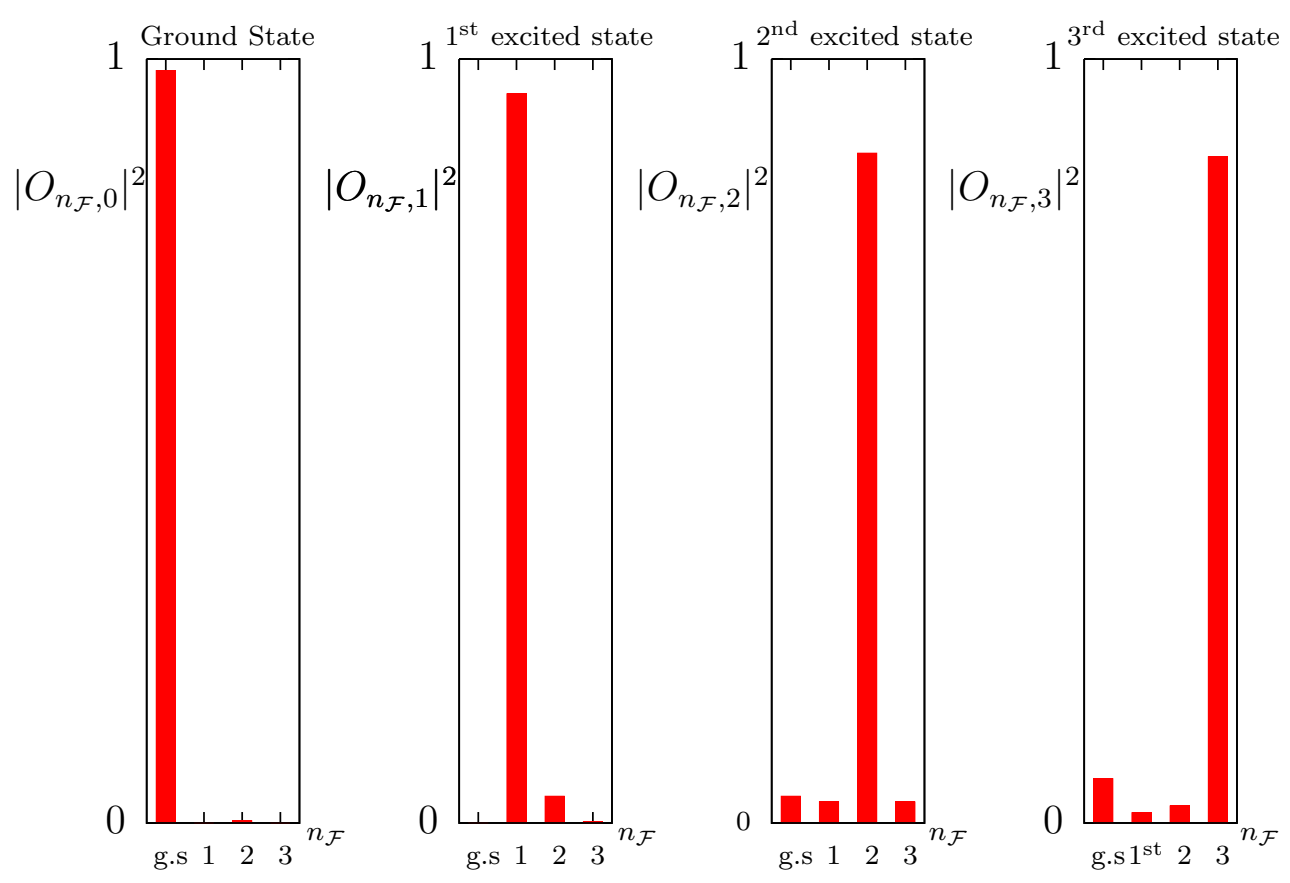

Figure 15: The Overlaps defined in Eq.(29) for the lightest four states in the $S U(5) k=2$ antisymmetric representation with $P=+, q=0$ and flux tube length $l=32 a$.

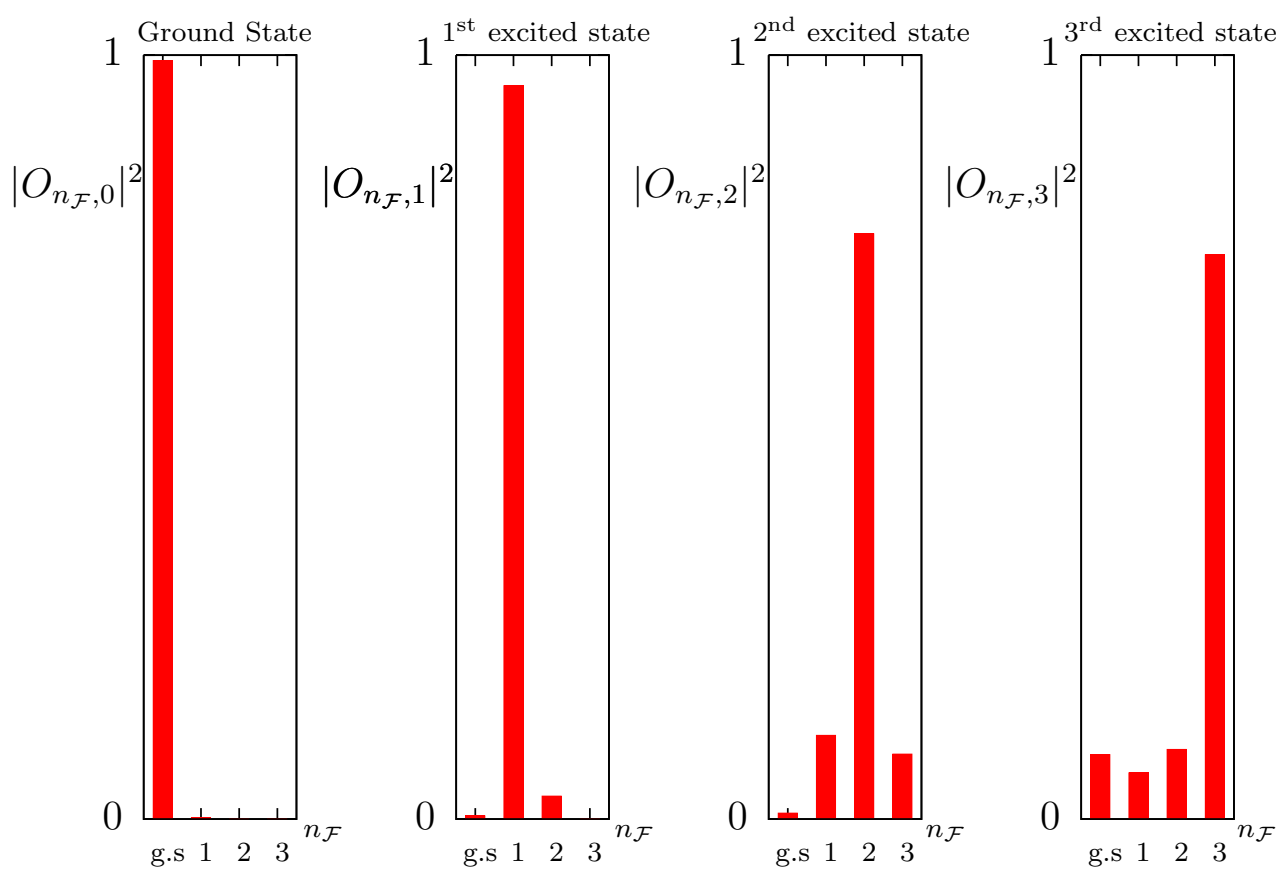

Figure 16: The Overlaps defined in Eq.(29) for the lightest four states in the $S U(5) k=2$ antisymmetric representation with $P=+, q=0$ and flux tube length $l=16 a$. 